MITSUBISHI ELECTRIC RESEARCH LABORATORIES

http://www.merl.com

\title{
Control Strategy for Long-Term Station-Keeping on Near-Rectilinear Halo Orbits
}

\author{
Muralidharan, Vivek; Weiss, Avishai; Kalabic, Uros \\ TR2020-006 January 08, 2020
}

\begin{abstract}
This work considers the control of a spacecraft in indefinite near-rectilinear halo orbit about the Earth-Moon L2. For indefinite station-keeping, it is important to minimize fuel consumption, while allowing for occasional transfer to a new orbit. The control scheme therefore consists of two components: the first component is the tracking of the nominal NRHO and the second component is an orbit correction maneuver between NRHO trajectories. The nominal NRHO is computed using a multiple-shooting technique that takes into account all forces on the spacecraft whose magnitude is larger than the dominant disturbance forces caused by navigational error. The tracking component is a linear-quadratic regulation scheme that rejects disturbances caused by orbit determination error, using a Lyapunov sublevel set that models the state covariance generated using sequential Kalman filter. The orbit correction maneuver is computed to minimize fuel costs.
\end{abstract}

AAS/AIAA Space Flight Mechanics Meeting

This work may not be copied or reproduced in whole or in part for any commercial purpose. Permission to copy in whole or in part without payment of fee is granted for nonprofit educational and research purposes provided that all such whole or partial copies include the following: a notice that such copying is by permission of Mitsubishi Electric Research Laboratories, Inc.; an acknowledgment of the authors and individual contributions to the work; and all applicable portions of the copyright notice. Copying, reproduction, or republishing for any other purpose shall require a license with payment of fee to Mitsubishi Electric Research Laboratories, Inc. All rights reserved. 



\title{
Control Strategy for Long-Term Station-Keeping on Near-Rectilinear Halo Orbits*
}

\author{
Vivek Muralidharan ${ }^{\dagger}$ \\ Purdue University, West Lafayette, IN 47907 \\ Avishai Weiss ${ }^{\ddagger}$ and Uroš Kalabić ${ }^{\S}$ \\ Mitsubishi Electric Research Laboratories, Cambridge, MA 02139
}

\begin{abstract}
This work considers the control of a spacecraft in indefinite near-rectilinear halo orbit about the Earth-Moon L2. For indefinite station-keeping, it is important to minimize fuel consumption, while allowing for occasional transfer to a new orbit. The control scheme therefore consists of two components: the first component is the tracking of the nominal NRHO and the second component is an orbit correction maneuver between NRHO trajectories. The nominal NRHO is computed using a multiple-shooting technique that takes into account all forces on the spacecraft whose magnitude is larger than the dominant disturbance forces caused by navigational error. The tracking component is a linear-quadratic regulation scheme that rejects disturbances caused by orbit determination error, using a Lyapunov sublevel set that models the state covariance generated using sequential Kalman filter. The orbit correction maneuver is computed to minimize fuel costs.
\end{abstract}

\section{Nomenclature}

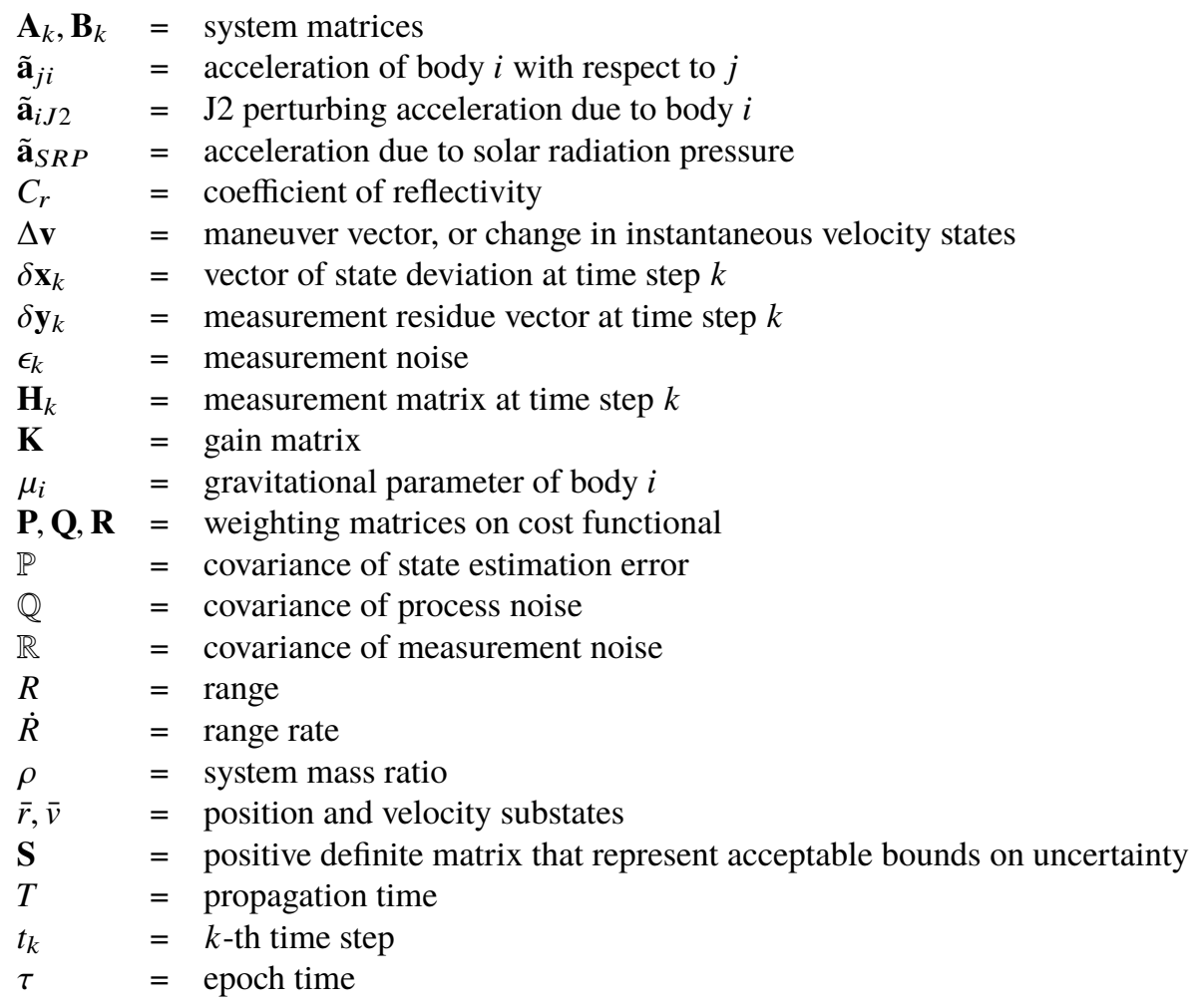

\footnotetext{
*This work was supported by Mitsubishi Electric Research Laboratories.

†Graduate Student, School of Aeronautics and Astronautics, muralidv@purdue.edu.

†rincipal Research Scientist, weiss@merl.com.

§esearch Scientist, kalabic@merl.com.
} 


$\begin{array}{ll}\mathbf{x} & =\text { state vector } \\ \hat{\mathbf{x}} & =\text { estimate of state vector } \\ \mathbf{w}_{k} & =\text { process noise }\end{array}$

\section{Introduction}

Near-rectilinear halo orbits (NRHOs) are closed trajectories around the L1 or L2 libration points in restricted three-body systems, whose stability indices are stable or close-to-stable. Due to their stability characteristics, they are potentially useful for the placement of lunar gateways for deep-space exploration [1]. In reality, NRHOs are never stable due to the effect of forces of other celestial bodies not considered in the restricted three-body problem, such as gravitational forces from other celestial bodies, solar radiation, magnetic forces and, not least of all, navigational errors and hardware limitations. All of these phenomena combine to destabilize the nominal orbit, therefore requiring the development of active control techniques to ensure that a spacecraft remains on the desired trajectory.

In this work, we propose a systematic design of controllers for indefinite station-keeping on NRHOs about the Earth-Moon L2 libration point. The design consist of three parts: firstly, the determination of a zero-cost trajectory which is close to an NRHO obtained as solution to the circular restricted three-body problem (CR3BP), and which takes into account all major, predictable disturbance forces; secondly, the design of a station-keeping technique that stabilizes the computed trajectory and is effective at controlling the spacecraft in the presence of unpredictable disturbance forces; and finally, low fuel cost transfer from one trejectory to another.

This systematic approach aims to achieve low fuel costs in station-keeping. By considering major, predictable disturbance forces in the computation of a zero-cost trajectory, we reduce the problem of station-keeping to that of rejecting unpredictable and predictable-but-negligible disturbance forces. The definition of a major predictable disturbance is one whose magnitude is larger than that of the largest unpredictable disturbance. This is consistent with our stated goal of lowering fuel costs as much as possible since we consider directly in computation all disturbances that must be considered in order to lower fuel costs: considering unpredictable disturbance is not feasible by definition and disturbances with force magnitudes lower than that of unpredictable forces are therefore negligible.

Our force-magnitude analysis is performed by computing an NRHO using Earth, Moon and Sun ephemeris data for knowledge of the gravitational pulls of these bodies, and then measuring the gravitational forces of other planets in the solar system as well as the force from solar radiation pressure and $\mathbf{J} 2$ zonal harmonics. According to our analysis, we determine that, apart from the gravitational forces of the Earth, Moon and Sun, the major, predictable forces acting on the spacecraft are that of solar radiation pressure (SRP) and Moon J2 on some orbits. All other predictable forces are less than the dominant unpredictable force: the indirect disturbance force caused by navigation error on the controller [2]. We quantify navigation error by assuming that the spacecraft position and velocity is obtained from the Deep Space Network (DSN). DSN measurement errors are quantifiable [3] and can be determined for spacecraft in NRHO [4]. The result of the quantification is a normally-distributed error profile whose uncertainty is directly correlated with the magnitude of the disturbance force resulting from navigational error.

We compute an NRHO orbit using a multiple-shooting technique that incorporates the ephemeris data of the Earth, Moon and Sun to determine the gravitational forces of those bodies as well as the SRP and J2 perturbing accelerations. As is common in the literature [5], we begin with an NRHO trajectory in the Earth-Moon CR3BP as an initial guess to our multiple-shooting algorithm and compute a solution corresponding to a solution where external forces are a fraction of their true magnitude. We then repeat the computation, growing the magnitude of the external forces until we have computed our free trajectory. Although NRHOs are closed in the solution to the CR3BP, our trajectory is not closed because closed trajectories do not exist in the chaotic dynamics of space. Our interest is in long-horizon missions, where the spacecraft would stay in NRHO indefinitely, and this requires regularly changing orbits from one NRHO trajectory to the next.

Having determined an appropriate NRHO baseline trajectory, we design a station-keeping scheme for long-horizon, low-cost tracking. We propose a two-stage approach that seeks to minimize fuel costs over the lifetime of the mission. The stages consist of station-keeping on a free trajectory and performing orbit correction maneuver between trajectories. The fuel costs of the orbit correction maneuver, also called a long-horizon maneuver as in [6], are more than an order of magnitude larger than those of station-keeping. For this reason, we pursue a minimal-velocity approach in minimizing fuel costs during orbit correction maneuvers and linear-quadratic regulation (LQR) for control of spacecraft during station-keeping. This two-stage approach allows us to reasonably minimize fuel costs, while ensuring good control characteristics for the majority of the mission, when the spacecraft is tracking the free NRHO trajectory. 


\section{Mission Design}

\section{A. Determination of Major Forces}

To compute a free trajectory to the best of our abilities, we must include all forces whose effect can be modeled or predicted in the computation of the free trajectory and whose effect will be greater than noisy disturbance effects. The largest unpredictable, noisy disturbance is that of navigational error and therefore we wish to determine all predictable forces whose effect on the spacecraft is more dominant than navigational error. To do this, we first compute our desired NRHO as a solution to the CR3BP and then compare the magnitude of known forces that would act on the spacecraft as it stays in orbit.

The vast majority of the acceleration on the spacecraft $i$, with respect to the Earth-Moon barycenter $q$, is given by,

$$
\tilde{\mathbf{a}}_{q i}=\sum_{j} \tilde{\mathbf{a}}_{j i}+\tilde{\mathbf{a}}_{\text {srp }}
$$

where $\tilde{\mathbf{a}}_{j i}$ is the net perturbing acceleration due to the gravitational force of a celestial body $j$ and $\tilde{\mathbf{a}}_{\text {srp }}$ is the net perturbing acceleration due to solar radiation pressure. The net perturbing gravitational accelerations due to the Earth $e$ and the Moon $m$ with respect to the Earth-Moon barycenter are given by,

$$
\begin{aligned}
\tilde{\mathbf{a}}_{e i} & =-\frac{\mu_{e}+(1-\rho) \mu_{i}}{r_{e i}^{3}} \mathbf{r}_{e i}+\tilde{\mathbf{a}}_{e, \mathrm{~J} 2}, \\
\tilde{\mathbf{a}}_{m i} & =-\frac{\mu_{m}+\rho \mu_{i}}{r_{m i}^{3}} \mathbf{r}_{m i}+\tilde{\mathbf{a}}_{m, \mathrm{~J} 2},
\end{aligned}
$$

where $\mu_{e}$ and $\mu_{m}$ are the Earth and Moon gravitational parameters, respectively, $\rho=\frac{\mu_{m}}{\mu_{e}+\mu_{m}}$ is the mass ratio of the Earth-Moon system, $\mathbf{r}_{j k}$ is the displacement from body $j$ to body $k$, and $\tilde{\mathbf{a}}_{e, \mathrm{~J} 2}$ and $\tilde{\mathbf{a}}_{m, \mathrm{~J} 2}$ represent the $\mathrm{J} 2$ perturbing accelerations due to Earth and Moon, respectively. The net perturbing gravitational accelerations due to the other celestial bodies are given by,

$$
\tilde{\mathbf{a}}_{j i}=\mu_{j}\left(\frac{\mathbf{r}_{j i}}{r_{j i}^{3}}-(1-\rho) \frac{\mathbf{r}_{j e}}{r_{j e}^{3}}-\rho \frac{\mathbf{r}_{j m}}{r_{j m}^{3}}\right),
$$

and the net perturbing acceleration due to solar radiation pressure is,

$$
\tilde{\mathbf{a}}_{\mathrm{SRP}}=a_{\mathrm{SRP}} \mathbf{r}_{s i}-(1-\rho) a_{e, \mathrm{SRP}} \mathbf{r}_{s e}-\rho a_{m, \mathrm{SRP}} \mathbf{r}_{s m},
$$

where $a_{\mathrm{SRP}}$ is the magnitude of the acceleration on the spacecraft due to solar radiation pressure and $a_{e, \mathrm{SRP}}$ and $a_{m, \mathrm{SRP}}$ is the same were the spacecraft placed at the center of mass of the Earth or Moon. The magnitude of the components in Eq. 1. acting on a spacecraft along an NRHO of perilune radius of $4000 \mathrm{~km}$ are plotted in Figure 1 while on a halo orbit with perilune radius of $40000 \mathrm{~km}$ is plotted in Figure 2. To determine which of these forces have effects that are more dominant than navigation error, we apply each force separately to our NRHO trajectory and determine the resulting tracking error. With the initial condition set to the halo orbit injection (HOI) state, we simulate the dynamics with the effect of each force added to the Earth, Moon and Sun gravitational forces, with which the nominal trajectory was computed. The results corresponding to an NRHO with perilune radius of $4000 \mathrm{~km}$ and a halo orbit with perilune radius 40,000km are shown in Figure 3 and Figure 4 respectively, from which we can see that, apart from the Earth and the Moon, the dominant forces are the gravitational pull of the Sun and the force from SRP. Due to close proximity to the Moon, in the case of the NRHO, J2 perturbations are nontrivial and we therefore include $\mathrm{J} 2$ perturbing acceleration whenever we compute an NRHO. Since the net acceleration due to the solar radiation pressure remain fairly consistent across the halo family, we therefore include SRP when computing any orbit under consideration. A spacecraft with mass $15,000 \mathrm{~kg}$ and $16 \mathrm{~m}^{2}$ surface area facing the Sun is considered. Previously, Chang'e-5 used a coefficient of reflectivity $C_{r}=1.24$ [7]; the ARTEMIS mission [8] indicates $C_{r}$ ranging between 1.10 and 1.16. Consistent with literature on other Lunar missions, we choose $C_{r}=1.15$.

\section{B. Computation of NRHOs}

For the baseline trajectory, we compute a southern L2 NRHO with perilune radius of $4000 \mathrm{~km}$, which corresponds to a sidereal resonance of 4:1. The method that we use is the multiple-shooting technique described in [9]. Before computing a trajectory corresponding to the dominant forces, we begin by computing an NRHO in the Earth-Moon 


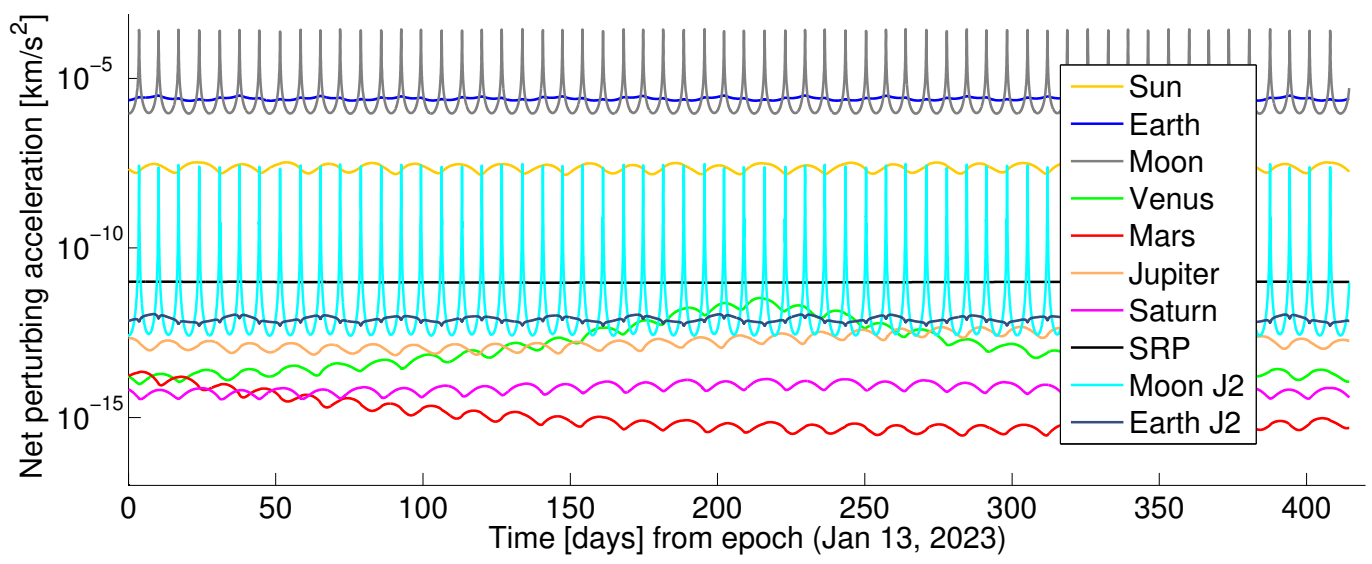

Fig. 1 Net perturbing acceleration due to various gravitational forces, solar radiation pressure, Earth and Moon J2 on NRHO with perilune radius $4000 \mathrm{~km}$

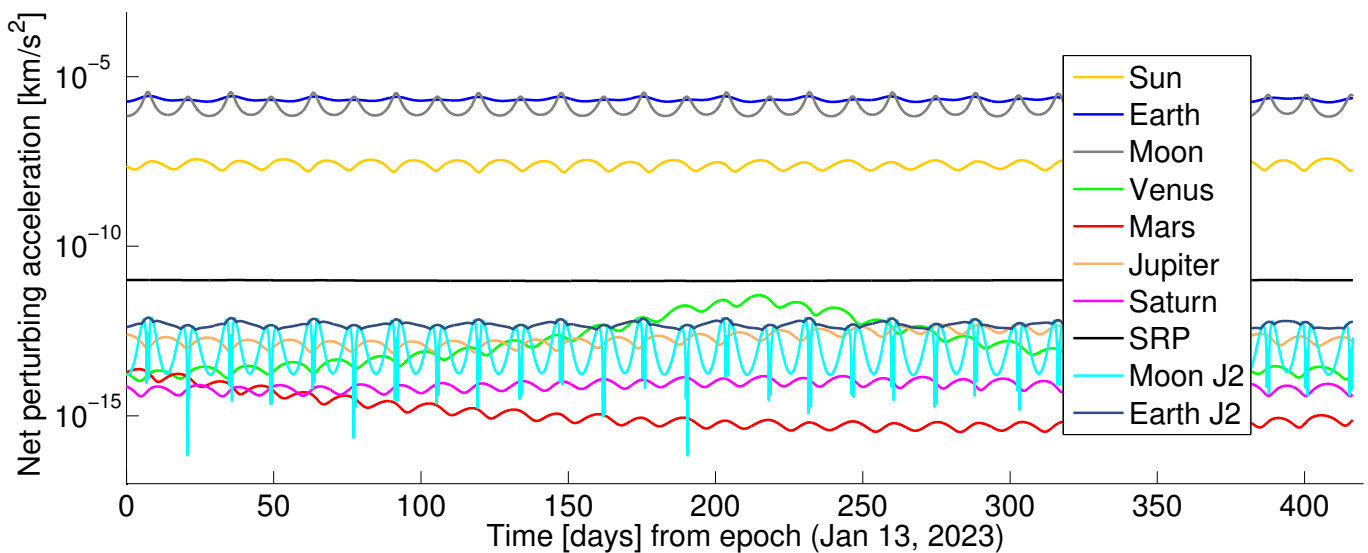

Fig. 2 Net perturbing acceleration due to various gravitational forces, solar radiation pressure, Earth and Moon $\mathrm{J} 2$ on halo orbit with perilune radius $40000 \mathrm{~km}$

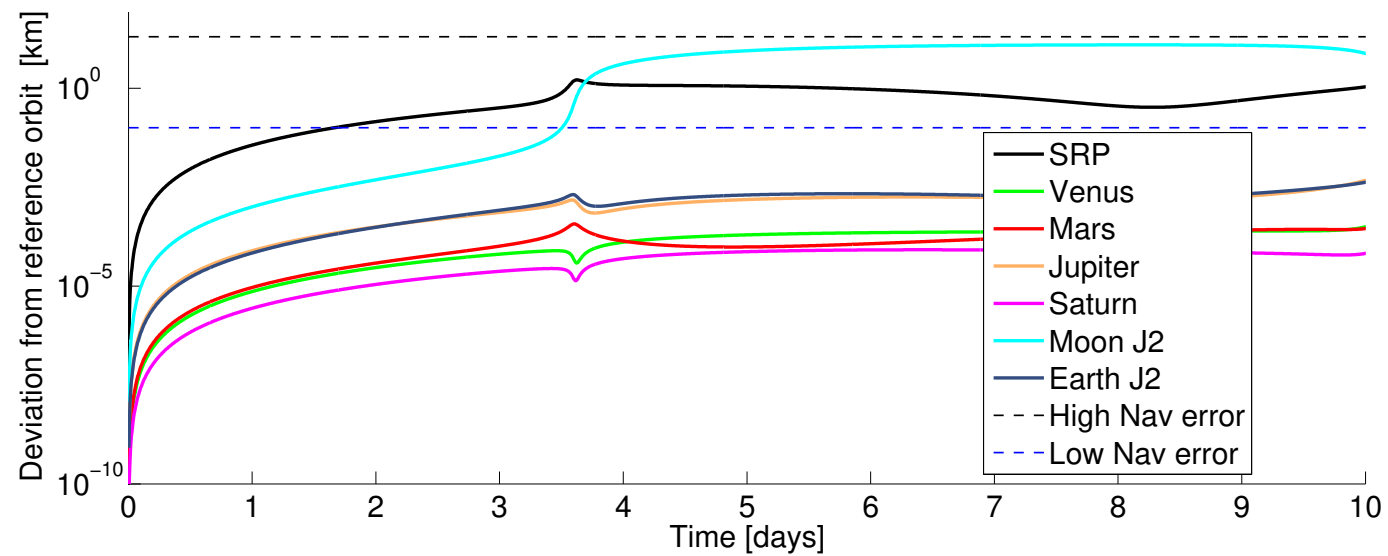

Fig. 3 Deviation from reference orbit due to various gravitational forces, solar radiation pressure, Earth and Moon J2 on NRHO with perilune radius $4000 \mathrm{~km}$

CR3BP model $\mathbf{x}^{\prime}$. Because the actual trajectory will not be periodic, we compute a long-horizon trajectory lasting over one year. We do this by concatenating 60 copies of the trajectory $\mathbf{x}^{\prime}$, subdivided into 20 arcs, and using this as an initial guess to the multiple-shooting scheme. We first compute $\mathbf{x}^{(0)}$, the trajectory corresponding to the forces determined from 


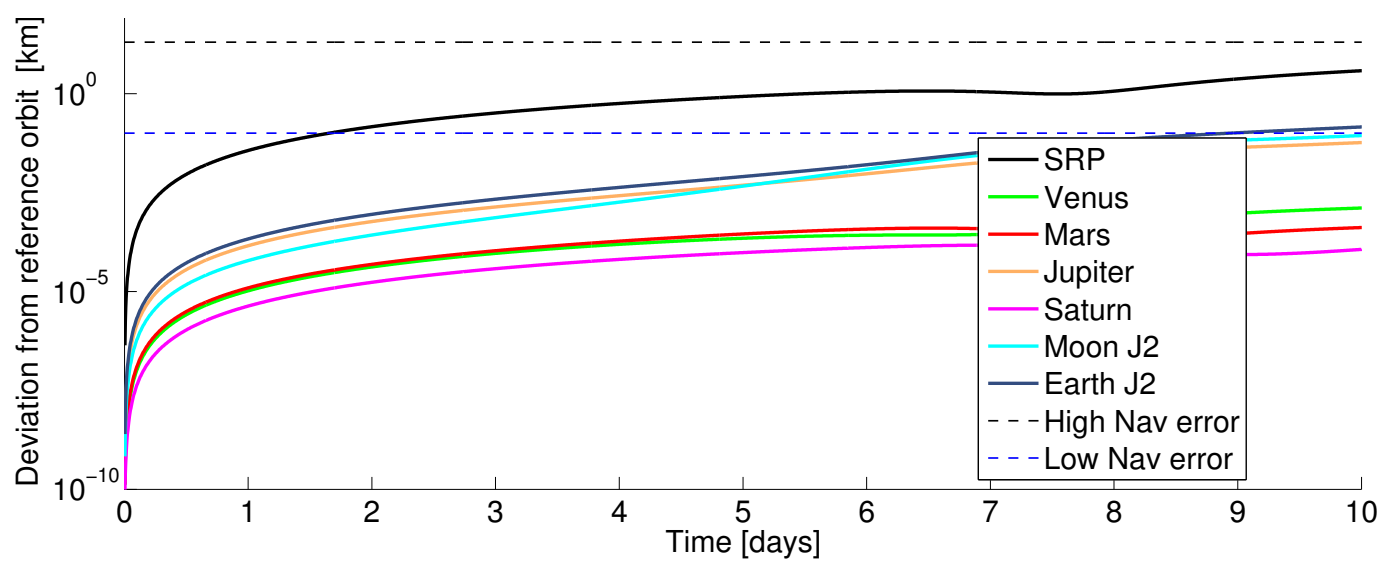

Fig. 4 Deviation from reference orbit due to various gravitational forces, solar radiation pressure, Earth and Moon J2 on halo orbit with perilune radius $40000 \mathrm{~km}$

the Earth and Moon ephemeris data. We then compute $\mathbf{x}^{(i)}, i=0,1,2, \ldots, 5$, where the $i$-th trajectory is computed with forces that are $0.2 i$ of the magnitude of the forces determined from the Sun ephemeris data. In this way, we compute multiple trajectories $\mathbf{x}_{k}$ for every year of the mission. Every trajectory $\mathbf{x}_{k}$ is computed using data from the $(k+1)$-th year of the mission.

\section{Orbit Determination Error}

The major contributor to the station-keeping cost is the uncertainty in tracking the position and velocity states of the spacecraft. The magnitude of uncertainty is proportional to the station-keeping cost. The tracking stations on the Earth take measurements in form of range, range rate, angular displacement, relative astronomical positions etc to determine the states of the spacecraft as accurately as possible. For a trajectory like the NRHO, where the spacecraft has a large change in $z$ position and a rapidly changing velocity in the co-normal direction, estimation of states becomes a challenge. Besides, the dynamical behavior of different regions along the orbit are contrasting. It may therefore not be sufficient to use a white noise with standard deviation along the position and velocity states to model the navigational uncertainty. A covariance matrix is thus propagated to measure uncertainty relative to the coordinates of the spacecraft. In this work, the range and range rate measurements are considered for computing the covariance. Uncertainty in the coordinates of the tracking station or any other random errors in determining mass of the celestial bodies, gravitational constant, etc are not taken into consideration.

The batch least squares filter and the sequential Kalman filter are two of the most commonly used filters for orbit determination. Orbit estimation, as early as the Apollo mission, has been performed using Kalman filter [10, 11]. Literature suggests that comparable results are produced using the batch least squares technique and the sequential Kalman filter [4, 12]. These techniques have been applied on the ARTEMIS mission that orbited the Earth-Moon L1 and L2 libration points for orbit determinations and station-keeping maneuver prediction [12]. Given the advantage of not having to store previously generated data and possibility of incorporating process noise makes sequential Kalman filtering a versatile tool [13] as well. For this application, a sequential Kalman filter has been implemented. The system we consider is given by,

$$
\begin{gathered}
\delta \mathbf{x}_{\kappa+1}=\mathbf{A}_{\kappa} \delta \mathbf{x}_{\kappa}+\mathbf{B}_{\kappa} \Delta \mathbf{v}_{\kappa}+\mathbf{w}_{\kappa} \\
\delta \mathbf{y}_{\kappa}=\mathbf{H}_{\kappa} \delta \mathbf{x}_{\kappa}+\boldsymbol{\epsilon}_{\kappa}
\end{gathered}
$$

where $\mathbf{A}_{\kappa}=\Phi\left(t_{\kappa+1}, t_{\kappa}\right), \mathbf{B}_{\kappa}$ is the sub-matrix of $\mathbf{A}_{\kappa}$ corresponding to the velocity components for control using impulsive maneuver, $\delta \mathbf{y}_{\kappa}$ is the measurement residue vector, $\delta \mathbf{y}_{\kappa}=[\delta R, \delta \dot{R}]^{T}$ where measurements are in range $R$ and range rate $\dot{R}$. The position and velocity state residue vector $\mathbf{x}_{K}=[\delta x, \delta y, \delta z, \delta \dot{x}, \delta \dot{y}, \delta \dot{z}]^{T}, \mathbf{w}_{\kappa}$ is the process noise such that $E\left[\mathbf{w}_{\kappa}\right]=0$ and $E\left[\mathbf{w}_{\kappa} \mathbf{w}_{\kappa}^{T}\right]=\mathbb{Q}, \mathbf{H}_{\kappa}$ is the measurement matrix and $\boldsymbol{\epsilon}_{\kappa}$ is the measurement noise. Since tracking stations are on the Earth, the range and range rate values are determined according to,

$$
R=\sqrt{(x+\mu)^{2}+y^{2}+z^{2}}
$$


Table 1 Orbit determination errors corresponding to different missions

\begin{tabular}{|c|c|c|c|c|}
\hline Range $(1 \sigma)$ & Range rate $(1 \sigma)$ & Mission type & Year & Reference \\
\hline $15.24 \mathrm{~m}$ & $15.24 \mathrm{~cm} / \mathrm{s}$ & Apollo & 1963 & Ref. [17 \\
\hline $15 \mathrm{~m}$ & $3 \mathrm{~mm} / \mathrm{s}$ & SOHO & 1992 & Ref. [4 \\
\hline $3 \mathrm{~m}$ & $0.3 \mathrm{~mm} / \mathrm{s}$ & Low Lunar Orbit & 1997 & Ref. 18 \\
\hline $10 \mathrm{~m}$ & $1 \mathrm{~mm} / \mathrm{s}$ & LRO & 2012 & Ref. $[19$ \\
\hline $10 \mathrm{~m}$ & $\begin{array}{c}3 \mathrm{~mm} / \mathrm{s} \text { at USN \& White Sands } \\
1 \mathrm{~mm} / \mathrm{s} \text { at DSN }\end{array}$ & LRO & 2014 & Ref. 20 \\
\hline $3 \mathrm{~m}$ & $1 \mathrm{~cm} / \mathrm{s}$ & Chang'E & 2019 & Ref. 21 \\
\hline $10 \mathrm{~m}$ & $1 \mathrm{~mm} / \mathrm{s}$ & - & & This work \\
\hline
\end{tabular}

$$
\dot{R}=\frac{(x+\mu) \dot{x}+y \dot{y}+z \dot{z}}{R}
$$

as a result of which the Jacobian matrix is given by $\mathbf{H}_{\kappa}(i, j)=\frac{\partial \mathbf{y}_{i}}{\partial \mathbf{x}_{j}}$. The measurement noise is assumed to be white with $E\left[\boldsymbol{\epsilon}_{\kappa}\right]=0$ and $E\left[\boldsymbol{\epsilon}_{\kappa, i} \boldsymbol{\epsilon}_{\kappa, j}\right]=\sigma_{i} \sigma_{j} \delta_{i j}=\mathbb{R}_{\kappa}$. For orbit estimation along a free trajectory $\Delta \mathbf{v}_{\kappa}=0$ in the process. Let the covariance in the estimation of state be

$$
\mathbb{P}_{\kappa \mid \kappa-1}=E\left[\left(\mathbf{x}_{K}-\hat{\mathbf{x}}_{K}\right)\left(\mathbf{x}_{K}-\hat{\mathbf{x}}_{K}\right)^{T}\right]
$$

where $\hat{\mathbf{x}}_{\kappa}$ be the estimate of $\mathbf{x}_{\kappa}$ computed after $\kappa-1$-th reading is observed. While,

$$
\mathbb{P}_{\kappa \mid \kappa}=E\left[\left(\mathbf{x}_{K}-\tilde{\mathbf{x}}_{K}\right)\left(\mathbf{x}_{K}-\tilde{\mathbf{x}}_{K}\right)^{T}\right]
$$

where $\tilde{\mathbf{x}}_{\kappa}$ be the estimate of $\mathbf{x}_{\kappa}$ computed after $\kappa$-th reading is available.

$$
\mathbb{P}_{\kappa \mid \kappa-1}=\mathbf{A}_{\kappa-1} \mathbb{P}_{\kappa-1 \mid \kappa-1} \mathbf{A}_{\kappa-1}^{T}+\mathbb{Q}
$$

With updated observation,

$$
\mathbb{P}_{\kappa \mid \kappa}=\left(\mathbb{P}_{\kappa \mid \kappa-1}^{-1}+\mathbf{H}_{\kappa}^{T} \mathbb{R}_{\kappa}^{-1} \mathbf{H}_{\kappa}\right)^{-1}
$$

Without a priori information, we set the initial value $\mathbb{P}_{\kappa \mid \kappa-1}$ to be very large. We then perform updates with additional data in an iterative process using Eq. 9 [4, 14]. Previously used values and in-use capabilities of tracking station across the globe are listed in Table 1 along with references. Consistent with those values, we considered a 1-sigma range measurement error of $10 \mathrm{~m}$ and 1-sigma range rate measurement error of $1 \mathrm{~mm} / \mathrm{s}$ in this work. Estimation is dependent on the number of observations available and the time interval over which observations are procured. Large numbers of observations provide better confidence at the cost of large computational time, while too few provide poor estimates. Based on heuristics, we determined that observations at intervals of 4 hours provided reliable estimates. We also assumed values of process noise in position and velocity states to be mean 0 with 1-sigma standard deviation of 1 $\mathrm{km}$ and $1 \mathrm{~cm} / \mathrm{s}$; this is consistent with literature reporting on prior Earth-Moon Lagrange point missions [15, 16].

\section{Station-Keeping}

\section{Orbit Maintenance}

Linear terms dominate the dynamical behavior in the neighborhood of the nominal trajectory $\mathbf{x}_{0}$. It is therefore appropriate to compute an optimal control policy using linearized dynamics. After discretization, the linear dynamics are given by,

$$
\delta \mathbf{x}_{k+1}=\mathbf{A}_{k} \delta \mathbf{x}_{k}+\mathbf{B}_{k} \Delta \mathbf{v}_{k},
$$

where $\delta \mathbf{x}_{k}=\mathbf{x}\left(t_{k}\right)-\mathbf{x}_{0}\left(t_{k}\right)+o\left(\delta \mathbf{x}_{k}\right), \mathbf{x}\left(t_{k}\right)$ is the true trajectory, $t_{k}$ is the discretization time-step, and $\Delta \mathbf{v}_{k}$ is the three-dimensional vector of instantaneous velocity change at $t_{k}$. In station-keeping, we aim to minimize the cost functional,

$$
\delta \mathbf{x}_{N}^{\mathrm{T}} \mathbf{P}_{N} \delta \mathbf{x}_{N}+\sum_{k=0}^{N-1} \delta \mathbf{x}_{k}^{\mathrm{T}} \mathbf{Q}_{k} \delta \mathbf{x}_{k}+\Delta \mathbf{v}_{k}^{\mathrm{T}} \mathbf{R}_{k} \Delta \mathbf{v}_{k}
$$


over $\left[t_{0}, t_{N}\right]$, which is the duration of the free trajectory $\mathbf{x}_{0}$. The solution is given by [22],

$$
\Delta \mathbf{v}_{k}=-\mathbf{K}_{k} \delta \mathbf{x}_{k},
$$

where the time-dependent gain $\mathbf{K}_{k}$ satisfies,

$$
\mathbf{K}_{k}=\left(\mathbf{R}_{k}+\mathbf{B}_{k}^{\mathrm{T}} \mathbf{P}_{k+1} \mathbf{B}_{k}\right)^{-1} \mathbf{B}_{k}^{\mathrm{T}} \mathbf{P}_{k+1} \mathbf{A}_{k},
$$

and $\mathbf{P}_{k}$ satisfies the Riccati difference equation,

$$
\mathbf{P}_{k}=\mathbf{Q}_{k}+\mathbf{A}_{k}^{\mathrm{T}} \mathbf{P}_{k+1} \mathbf{A}_{k}-\mathbf{A}_{k}^{\mathrm{T}} \mathbf{P}_{k+1} \mathbf{B}_{k} \mathbf{K}_{k}
$$

for $k=0, \ldots, N-1$.

Our goal is to use not much more energy than is needed in staying within this set of acceptable bounds and we take a systematic approach in determining the matrices $\mathbf{P}_{k}$. Let $\mathcal{P}_{k}(c)=\left\{\delta \mathbf{x}: \delta \mathbf{x}^{\mathrm{T}} \mathbf{P}_{k} \delta \mathbf{x} \leq c\right\}$ be the sublevel set corresponding to the matrix $\mathbf{P}_{k}$. The sublevel set satisfies the property that $\delta \mathbf{x}_{k} \in \mathcal{P}_{k}(c) \Longrightarrow \delta \mathbf{x}_{i} \in \mathcal{P}_{i}(c)$ for any $0 \leq i \leq k \leq N$. Let $\mathbf{S}$ be a positive definite matrix whose sublevel set is given by $\mathcal{S}(c)=\left\{\delta \mathbf{x}: \delta \mathbf{x}^{\mathrm{T}} \mathbf{S} \delta \mathbf{x} \leq c\right\}$. If $\mathbf{P}_{k} \geq \mathbf{S}$, then $\delta \mathbf{x} \in \mathcal{P}_{k}(c) \Longrightarrow \delta \mathbf{x} \in \mathcal{S}(c)$. The set $\mathcal{S}(c)$ represents the acceptable bounds on our tracking error. We can solve for the optimal solution by formulating a matrix optimization problem. We do this by letting $\tilde{\mathbf{P}}_{k}=\mathbf{P}_{k}-\mathbf{S}$, to obtain,

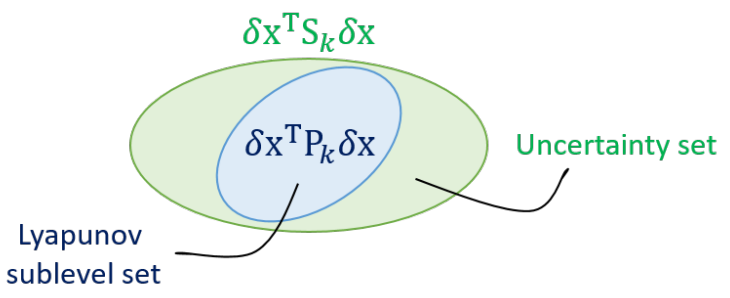

Fig. 5 Lyapunov set and uncertainty set

$$
\tilde{\mathbf{P}}_{k}+\mathbf{S}=\mathbf{A}_{k}^{\mathrm{T}}\left(\tilde{\mathbf{P}}_{k+1}+\mathbf{S}\right) \mathbf{A}_{k}-\mathbf{A}_{k}^{\mathrm{T}}\left(\tilde{\mathbf{P}}_{k+1}+\mathbf{S}\right) \mathbf{B}_{k}\left(\mathbf{R}_{k}+\mathbf{B}_{k}^{\mathrm{T}}\left(\tilde{\mathbf{P}}_{k+1}+\mathbf{S}\right) \mathbf{B}_{k}\right)^{-1} \mathbf{B}_{k}^{\mathrm{T}}\left(\tilde{\mathbf{P}}_{k+1}+\mathbf{S}\right) \mathbf{A}_{k},
$$

which after rearranging becomes the Riccati difference equation,

$$
\tilde{\mathbf{P}}_{k}=\tilde{\mathbf{Q}}_{k}+\mathbf{A}_{k}^{\mathrm{T}} \tilde{\mathbf{P}}_{k+1} \mathbf{A}_{k}-\left(\mathbf{A}_{k}^{\mathrm{T}} \tilde{\mathbf{P}}_{k+1} \mathbf{B}_{k}+\tilde{\mathbf{N}}_{k}^{\mathrm{T}}\right)\left(\tilde{\mathbf{R}}_{k}+\mathbf{B}_{k}^{\mathrm{T}} \mathbf{P}_{k+1} \mathbf{B}_{k}\right)^{-1}\left(\mathbf{B}_{k}^{\mathrm{T}} \tilde{\mathbf{P}}_{k+1} \mathbf{A}_{k}+\tilde{\mathbf{N}}_{k}\right),
$$

where $\tilde{\mathbf{Q}}_{k}=\mathbf{Q}_{k}-\mathbf{S}+\mathbf{A}_{k}^{\mathrm{T}} \mathbf{S} \mathbf{A}_{k}, \tilde{\mathbf{R}}_{k}=\mathbf{R}_{k}+\mathbf{B}_{k}^{\mathrm{T}} \mathbf{S} \mathbf{B}_{k}$, and $\tilde{\mathbf{N}}_{k}=\mathbf{B}_{k}^{\mathrm{T}} \mathbf{S} \mathbf{A}_{k}$. To ensure the existence of a positive semi-definite solution for the sequence of matrices $\left\{\tilde{\mathbf{P}}_{k}\right\}$, it is sufficient that the matrix,

$$
\left[\begin{array}{cc}
\tilde{\mathbf{Q}}_{k} & \tilde{\mathbf{N}}_{k} \\
\tilde{\mathbf{N}}_{k}^{\mathrm{T}} & \tilde{\mathbf{R}}_{k}
\end{array}\right],
$$

is positive semi-definite for all $k$, which is true if and only if its Schur complement,

$$
\tilde{\mathbf{Q}}_{k}-\tilde{\mathbf{N}}_{k}^{\mathrm{T}} \tilde{\mathbf{R}}_{k}^{-1} \tilde{\mathbf{N}}_{k}=\mathbf{Q}_{k}+\mathbf{A}_{k}^{\mathrm{T}} \mathbf{S} \mathbf{A}_{k}-\mathbf{S}-\tilde{\mathbf{N}}_{k}^{\mathrm{T}} \tilde{\mathbf{R}}_{k}^{-1} \tilde{\mathbf{N}}_{k},
$$

is positive semi-definite. To ensure that this is the case, we set $\mathbf{Q}_{k}$ to be the smallest semi-positive definite matrix which ensures the semi-positive definiteness of Eq.16, i.e., we set $\mathbf{Q}_{k}=-\mathbf{L}_{k}$, where $\mathbf{L}_{k}$ is the negative semi-definite component of $\mathbf{A}_{k}^{\mathrm{T}} \mathbf{S} \mathbf{A}_{k}-\mathbf{S}-\tilde{\mathbf{N}}_{k}^{\mathrm{T}} \tilde{\mathbf{R}}_{k}^{-1} \tilde{\mathbf{N}}_{k}$.

The penalty on control is chosen to be constant and equal in all directions, giving $\mathbf{R}_{k} \equiv r \mathbf{I}_{3}$ for some large scalar $r$. We solve Eq. 15 backwards-in-time with the final condition $\tilde{\mathbf{P}}_{N}=\mathbf{0}$. The minimum-energy solution is obtained by finding the solution for $r \rightarrow \infty$; however, practically and computationally, $r$ only need be a relatively large number.

\section{E. Coupled orbit estimation and maneuver generation}

The LQR gains are computed by solving the Riccati equation backwards in time, i.e., in the direction of stability; the covariance matrices, upon which the LQR weights are based, are computed by propagating the stable Kalman filter forwards in time. We therefore implement the algorithm, of Figure 6 to simultaneously solve for both. In the algorithm, we alternate iteratation in one direction and the other until convergence; convergence occurs relatively quickly, after about five iterations. A shown in Figure 7, station-keeping maneuvers and observations do not general occur at the same 
time. In the figure, we show that observations and estimate updates are performed at time instants $t_{k-1}^{i}$, corresponding to the $i$-th observation after the $(k-1)$-th control maneuver where, as stated previously, the difference between $t_{k-1}^{i}$ and $t_{k-1}^{i+1}$ is held constant at 4 hours by design. Similarly, the number of maneuvers over one time-period is also a design choice. We assume no thruster error, i.e., the value of maneuver determined by the controller is same as the maneuver implemented. Nevertheless, we note that the estimation of states using a Kalman filter can however take into consideration any error in maneuver execution by updating the value of $\mathbb{Q}$ matrix to include terms that relate to error in maneuver execution $\tilde{\mathbb{Q}}=\mathbb{Q}+\mathbf{B} E\left[\Delta \mathbf{v} \Delta \mathbf{v}^{\mathrm{T}}\right] \mathbf{B}^{\mathrm{T}}$. If a tracking observation does not exist at the maneuver location, e.g., $t_{k-1}^{n} \neq t_{k}$, the covariance matrix is propagated to the maneuver location $t_{k}$ after considering all readings until $t_{k-1}^{n}$ using Eq. (8). However, if $t_{k-1}^{n}=t_{k}$, the covariance matrix $\mathbb{P}$ is updated to include readings at $t_{k}$ using Eq. (9) before the control maneuver is determined. After a maneuver, the covariance at $t_{k}$ is used as an a prioiri input to the Kalman filter before sequential observations at $t_{k}^{1}, t_{k}^{2}$, and so on.

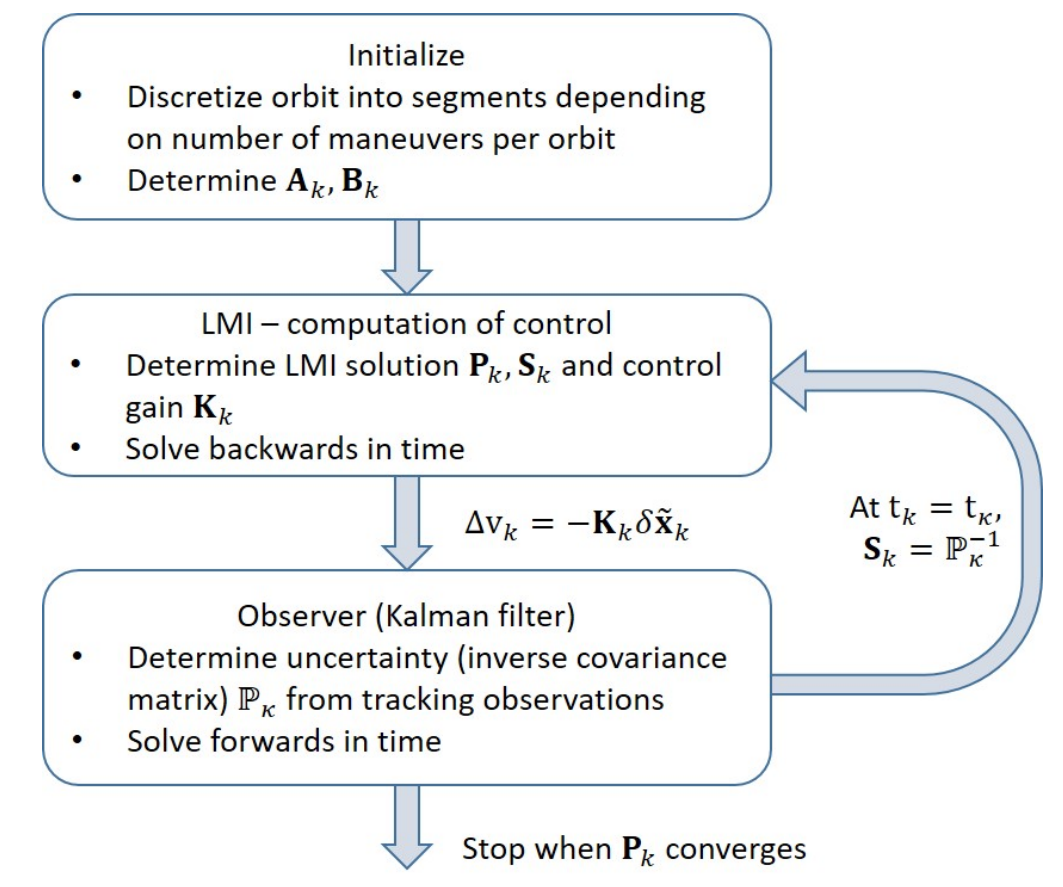

Fig. 6 Coupled algorithm for estimating maneuver size based on uncertainty set

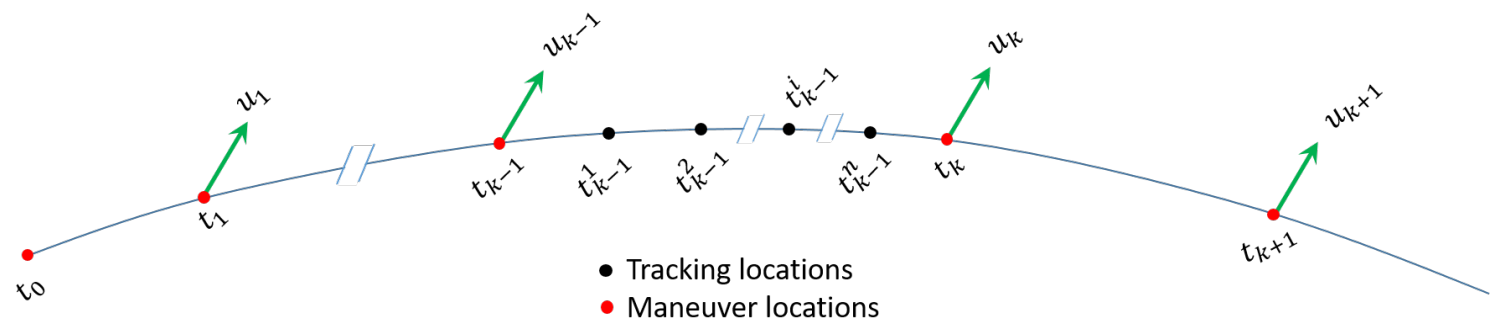

Fig. 7 Estimation and propagation of covariance from tracking observations

\section{F. Trajectory correction maneuvers}

It may not be desirable to remain on an NRHO indefinitely. For example, mission objectives may change depending on science constraints while on-orbit, requiring the spacecraft to change to a different reference path. Furthermore, numerical computation of a free trajectory for an indefinite duration is challenging and cumbersome and alternating 
between finite reference trajectories is a possible option. In either case, a trajectory correction maneuver may be required to transition the spacecraft from the current baseline trajectory to the new baseline trajectory. Since fuel available per mission is limited, it is desired to make the transition with the greatest possible fuel savings. With such a long-horizon maneuver [6], the spacecraft hops from one long horizon reference to another long-horizon reference once the maneuver is executed.

We propose a strategy to optimize total $\Delta \mathbf{v}$ using a two-maneuver scheme shown in Figure 8 . First, we implement a maneuver to depart from current/initial baseline to the transfer arc and then a second maneuver to hop from transfer arc onto the new baseline trajectory. The procedure can be extended to more than two maneuvers along the transfer arc. Define the cost function to be minimized as,

$$
J=J_{1}+J_{2}
$$

where,

$$
\begin{aligned}
& J_{1}=\sqrt{\left(\mathbf{v}_{1}^{a r r}-\mathbf{v}_{1}^{d e p}\right)^{\mathrm{T}}\left(\mathbf{v}_{1}^{a r r}-\mathbf{v}_{1}^{d e p}\right)}, \\
& J_{2}=\sqrt{\left(\mathbf{v}_{2}^{a r r}-\mathbf{v}_{2}^{d e p}\right)^{\mathrm{T}}\left(\mathbf{v}_{2}^{a r r}-\mathbf{v}_{2}^{d e p}\right)},
\end{aligned}
$$

and $J_{1}$ is the cost to depart from initial baseline onto the transfer arc while $J_{2}$ is the cost of placing the spacecraft onto the new baseline trajectory. Since the velocities on the baselines are fixed, the velocity on arrival on initial baseline $\mathbf{v}_{1}^{a r r}$ and departing velocity on the new baseline $\mathbf{v}_{2}^{d e p}$ are constants. To obtain a feasible free trajectory along the transfer arc, we need to satisfy continuity constraints on the position, velocity and time at any intermediate patch point.

Continuity can be enforced by dividing the transfer arc into $n$ patch points as shown in Figure 9 We then introduce the $(8 n-12)$-dimensional vector of design variables $\bar{X}=\left[\bar{v}_{1}, \bar{x}_{2}, \ldots, \bar{x}_{n-1}, T_{1}, \ldots, T_{n-1}, \tau_{2}, \ldots, \tau_{n-1}\right]^{\mathrm{T}}$. Note that the initial velocity is a variable that is to be optimized since it is the $\Delta \mathbf{v}$ required to place the spacecraft on a new trajectory. The $(7 n-10)$-dimensional constraint vector $\bar{F}(\bar{X})$ is defined as,

$$
\bar{F}(\bar{X})=\left[\begin{array}{c}
\bar{x}_{2}^{t}\left(\bar{x}_{1}\right)-\bar{x}_{2} \\
\bar{x}_{3}^{t}\left(\bar{x}_{2}\right)-\bar{x}_{3} \\
\vdots \\
\bar{r}_{n}^{t}\left(\bar{x}_{n-1}\right)-\bar{r}_{n} \\
\tau_{2}-\left(\tau_{1}+T_{1}\right) \\
\vdots \\
\tau_{n}-\left(\tau_{n-1}+T_{n-1}\right)
\end{array}\right],
$$

and must be satisfied for a feasible transfer. Here $\bar{x}_{i}^{t}\left(\bar{x}_{i-1}\right)$ is the resultant state near node $i$ as a result of propagation of states $\bar{x}_{i-1}$. Instead of enforcing velocity continuity at node $n$, optimize the by setting $\bar{v}_{n}^{t}\left(\bar{x}_{n-1}\right)=\mathbf{v}_{2}^{\text {arr }}$ and $\bar{v}_{n}=\mathbf{v}_{2}^{\text {dep }}$ and optimizing according to Eq. (19). For optimization, we use the numerical, interior-point-method-based solver IPOPT. Since the state transition matrix, the velocity and acceleration terms are analytically derivable quantities, we are able to improve the optimization by providing a user-defined gradient and Jacobian.

\section{Results}

\section{A. Effect of weighting matrix}

In this section, we present our orbit maintenance results using our LQR scheme. The control scheme design uses a choice of matrix $\mathbf{S}=\mathbb{P}^{-1}$ for the choice of the set of acceptable bounds, and the choice of weight $r$ was varied between $10^{12}$ to $10^{17}$ on the control input. To increase confidence in a choice, we performed 1000 Monte Carlo simulations, each one with navigational error generated from a different random seed. While the values of $\mathbf{S}, \mathbf{P}$ and $\mathbf{Q}$ are determined by the algorithm, the value of control penalty matrix $\mathbf{R}$ is a design variable. Sincei $t$ is assumed that the spacecraft can perform maneuvers in all the three directions independently without any difference in thruster capacity, equal weights were chosen for all three directions, i.e., $\mathbf{R}=\operatorname{diag}\left(r_{x}, r_{y}, r_{z}\right)$ with $r=r_{z}=r_{y}=r_{z}$. 


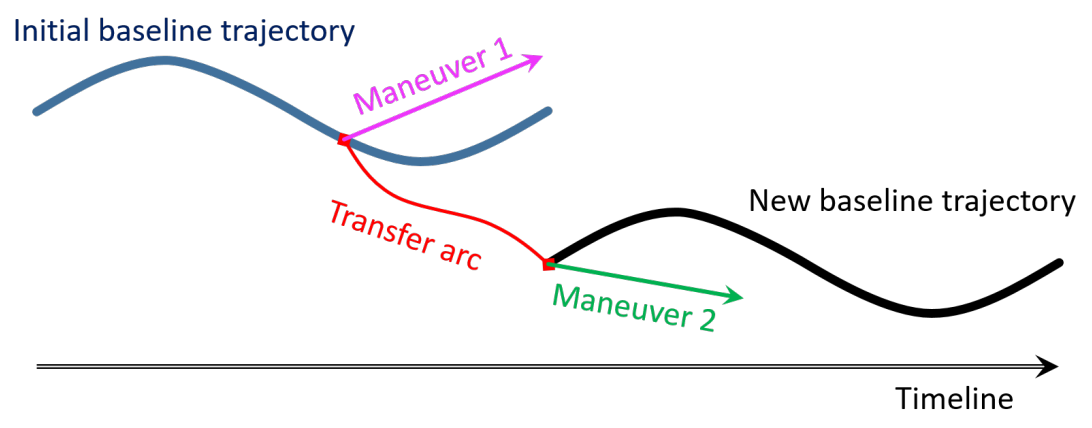

Fig. 8 Trajectory correction maneuver to transfer between baseline trajectories

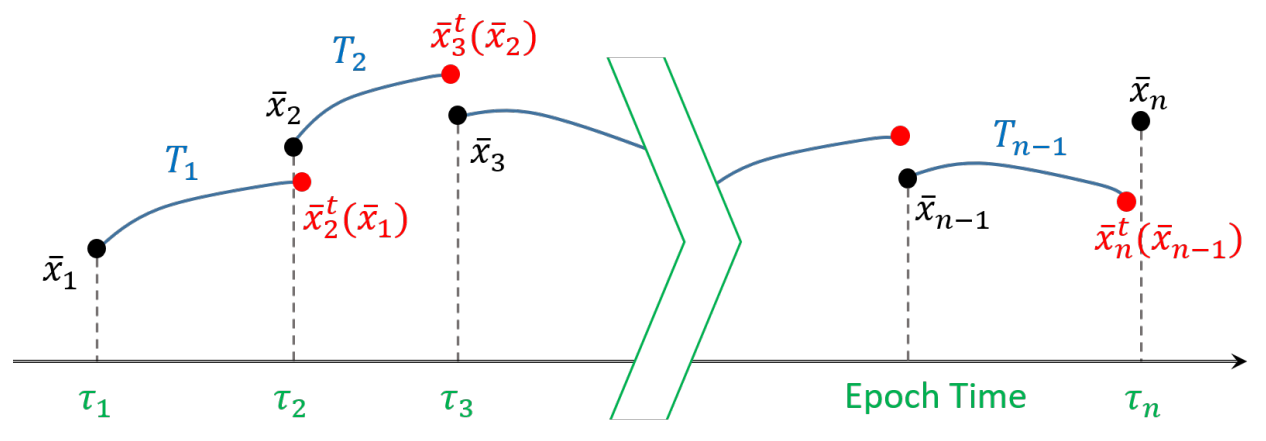

Fig. 9 Setup of optimization scheme

In Table 2, we present station-keeping costs for different choices of weight $r$ when performing a single maneuver at the apoapsis region for the NRHO with perilune radius $4000 \mathrm{~km}$. A Pareto optimal plot between mean annual cost and mean deviation is shown in Figure 10 and the results show that the minimum cost occurs when the penalty is set to $r=10^{17}$, but causes the largest deviation from the reference trajectory. Figures $11 \mathrm{a}$ and $11 \mathrm{~b}$ show the control history in terms of maneuver size and deviation from the reference trajectory for 1 year of simulation, respectively. Since maneuver magnitude is penalized severely, very low maneuver magnitude is generated by the controller, but this choice results in poor tracking of the spacecraft. In fact, the spacecraft departs away from the reference trajectory gradually over time. On the contrary, station-keeping costs for $r=10^{14}$ are marginally higher indicated in Figure 12a but the control history in terms of state deviation, according to Figure $12 \mathrm{~b}$ clearly show bounded behavior where the spacecraft states are well within $10 \mathrm{~km}$ from the baseline path. Lower choice of weight causes higher emphasis on close tracking, leading to higher fuel consumption; higher choice of weight results in less fuel consumption at the cost of poor tracking performance. Due to close tracking with a near minimal station-keeping cost, the value of $r=10^{14}$ is selected as the appropriate choice for the technique. It was also observed that this value of $r$ provides good results for other cases as well.

Table 2 Station-keeping cost for different choices of $r$ with 1 maneuver per orbit at apoapsis region for 4:1 sidereal resonant NRHO with perilune radius $4000 \mathrm{~km}$

\begin{tabular}{|c|c|c|}
\hline$r$ & Mean annual cost $(\mathrm{m} / \mathrm{s})$ & Mean deviation $[\mathrm{km}]$ \\
\hline $10^{12}$ & 0.253 & 2.923 \\
$10^{13}$ & 0.113 & 1.814 \\
$10^{14}$ & 0.083 & 1.596 \\
$10^{15}$ & 0.064 & 1.818 \\
$10^{16}$ & 0.052 & 3.497 \\
$10^{17}$ & 0.050 & 11.807 \\
\hline
\end{tabular}




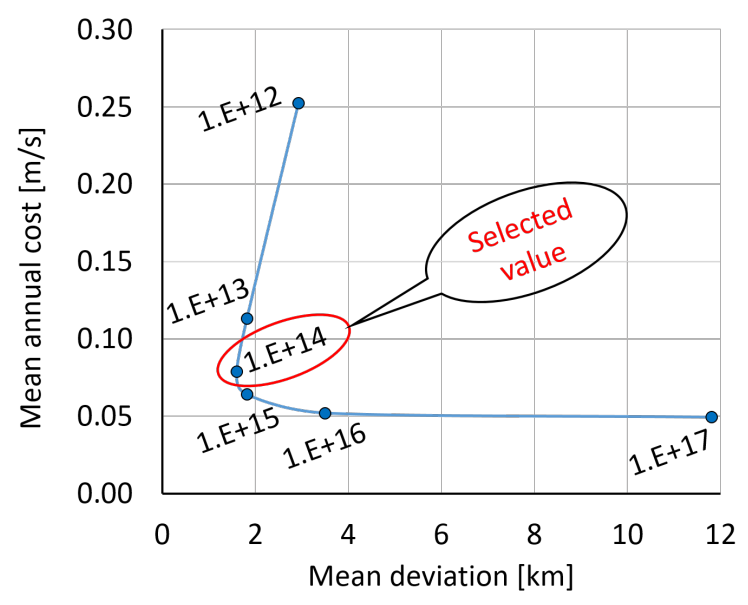

Fig. 10 Mean Annual cost vs deviation Pareto plot

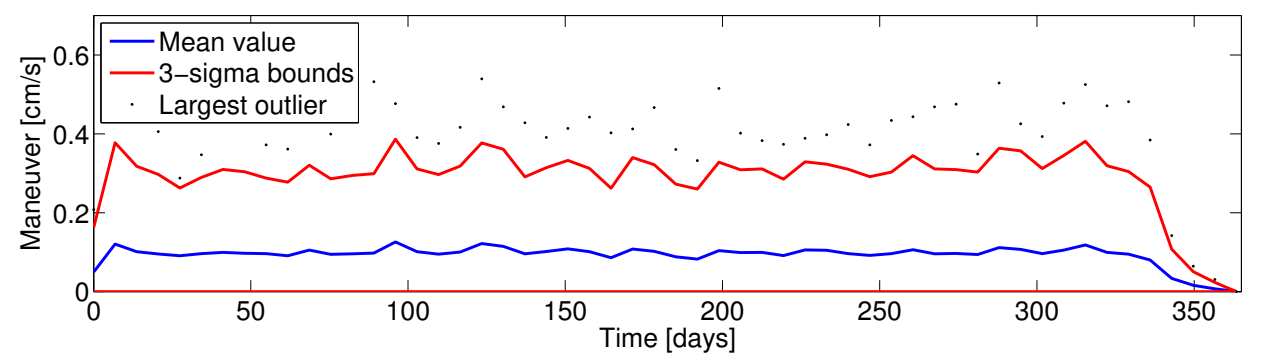

(a) Maneuver magnitude vs time

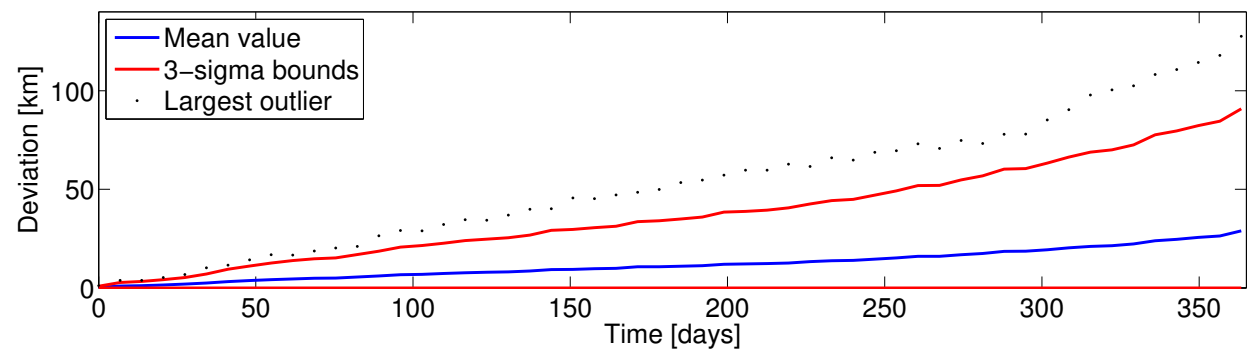

(b) Deviation vs time

Fig. 11 Maneuver magnitude and position deviation for NRHO with perilune radius $4000 \mathbf{~ k m}$ with $r=10^{17}$. Station-keeping maneuvers performed once per revolution at the apoapsis region.

\section{B. Cost along NRHO}

We perform analysis of the station-keeping method for both the stable NRHO as well as the unstable orbits of the halo family. Multiple maneuvers per revolution have been performed for each case. Specifically, for the stable NRHO a distinct behavior was observed for cases in which there exists a maneuver at the periapsis region versus without a maneuver there. Maneuver locations are equally spaced in time starting from the halo orbit injection location, consequently a maneuver location may or may not precisely coincide with the closest and farthest point from the moon on the trajectory, i.e., the periapsis or apoapsis. With the same intuition, here the periapsis and apoapsis region is defined with respect to the maneuver locations that is closest or farthest to the moon every revolution. This may not be precisely coincide to the periapsis or apoapsis on the actual trajectory. For the sample NRHOs tested here, Figure 13a presents the annual station-keeping cost for maneuver locations that are distinctly far from the periapsis region. First, a maneuver occurs in the apoapsis region and the successive maneuvers are equally spaced in times from there on. The cases corresponding to 0.25 or 0.5 maneuvers per revolution essentially mean that maneuvers happen at the 
apoapsis region once in 4 revolutions or 2 revolutions, respectively. In contrast, Figure $13 \mathrm{~b}$ presents the mean annual station-keeping cost for cases that include maneuvers at the periapsis region. The y-axis for both Figures $13 \mathrm{a}$ and $13 \mathrm{~b}$ are scaled equally for comparison. Having no maneuvers in the periapsis region tends to provide lower station-keeping costs than otherwise. We note that similar results are found in the literature [6]. For no maneuvers at the periapsis region, a minima in the station-keeping costs is observed for one maneuver per revolution at the apoapsis region. One maneuver every two or four revolutions are sufficient but increase the overall cost. Too few maneuvers allow ample time for the spacecraft to deviate away from the reference path, requiring a larger maneuver to control. Having an odd number of maneuvers starting at the apoapsis region ensures periapsis avoidance, yet having multiple maneuvers does not seem to decrease the orbit maintenance cost. For cases that include maneuvers in the periapsis region, the mean cost is significantly higher than cases without a maneuver at the periapsis region. Due to the high eccentricity of the NRHO orbits, the spacecraft comes in close proximity with the moon at the periapsis region. Due to the high non-linearity and instability in this region, an extremely high maneuver is generated that may be redundant, as the NRHO is close-to-stable. Having few but an even number of maneuvers provides sufficient time for the spacecraft to deviate away from the nominal path after the large maneuver generated at the periapsis region, and consequently a very high mean cost is estimated. Too large a number of even maneuvers attempts to overcome the instability caused at the periapsis, and perhaps may be redundant. Overall we determine that for NRHOs, a single maneuver at the apoapsis region is sufficient and the best condition for orbit maintenance. Note that, for NRHOs with a periapsis radius less than $3200 \mathrm{~km}$, the spacing between maneuvers is less than the tracking interval for orbit determination using sequential Kalman filter if 40 maneuvers per orbit are considered, hence these NRHOs were not selected for comparison.

\section{Cost along unstable halo}

Unstable halo orbits require a larger number of maneuvers for efficient station-keeping using this technique. As shown in Figure 14, unlike the NRHO where a single maneuver per revolutions is sufficient, station-keeping costs decrease with an increase in number of maneuvers per revolution to a certain value before stagnating and further increasing. For most of unstable halo orbits tested, a range of 5 to 16 maneuver per orbit seems to work best. Despite these orbits being unstable, the technique efficiently maintains the spacecraft in the vicinity for under $0.5 \mathrm{~m} / \mathrm{s}$ per year. For sufficiently large number of maneuvers a narrow belt of station-keeping cost is observed irrespective of the size of the halo orbit.

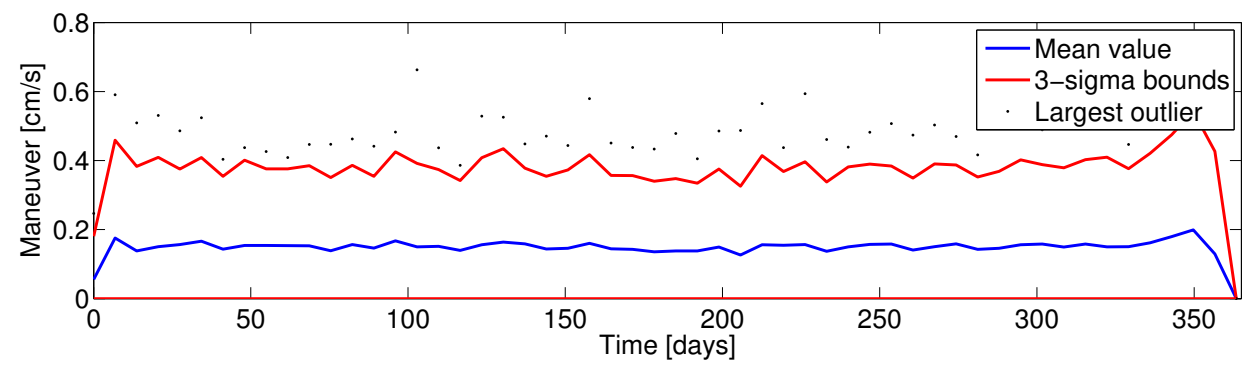

(a) Maneuver magnitude vs time

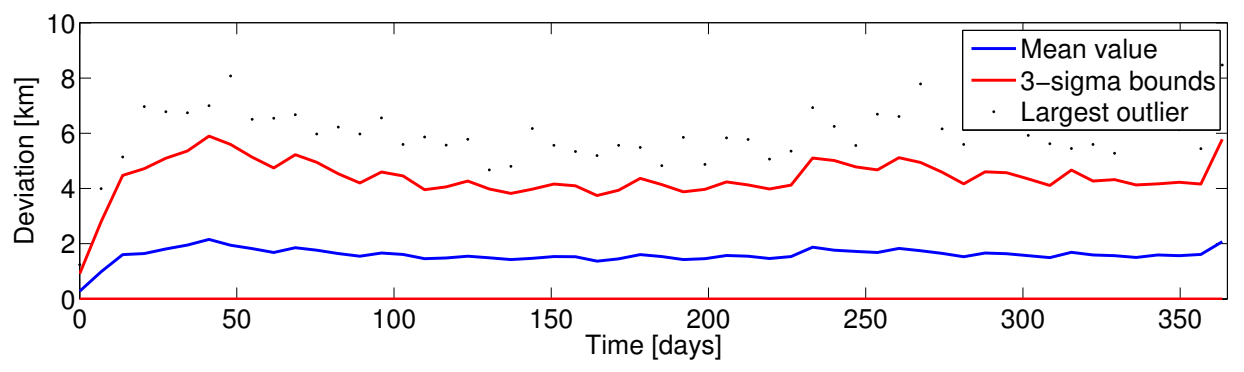

(b) Deviation vs time

Fig. 12 Maneuver magnitude and position deviation for NRHO with perilune radius $4000 \mathrm{~km}$ with $r=10^{14}$. Station-keeping maneuvers performed once per revolution at the apoapsis region. 


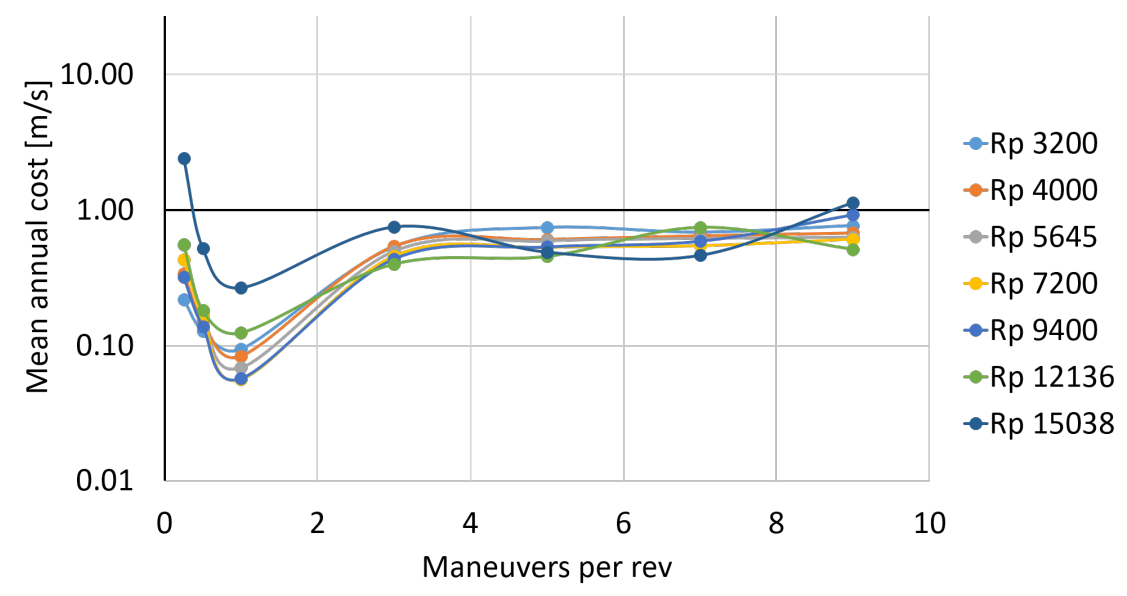

(a) No maneuvers at periapsis region

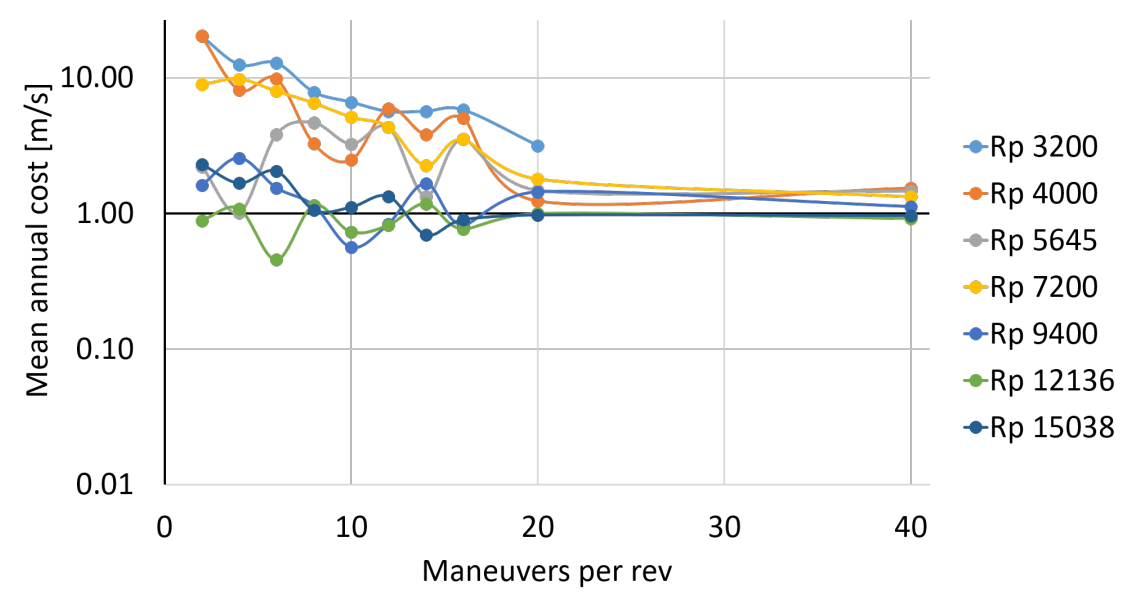

(b) Maneuvers included at periapsis region

Fig. 13 Mean annual cost for selected NRHO orbits with different number of maneuvers per revolution

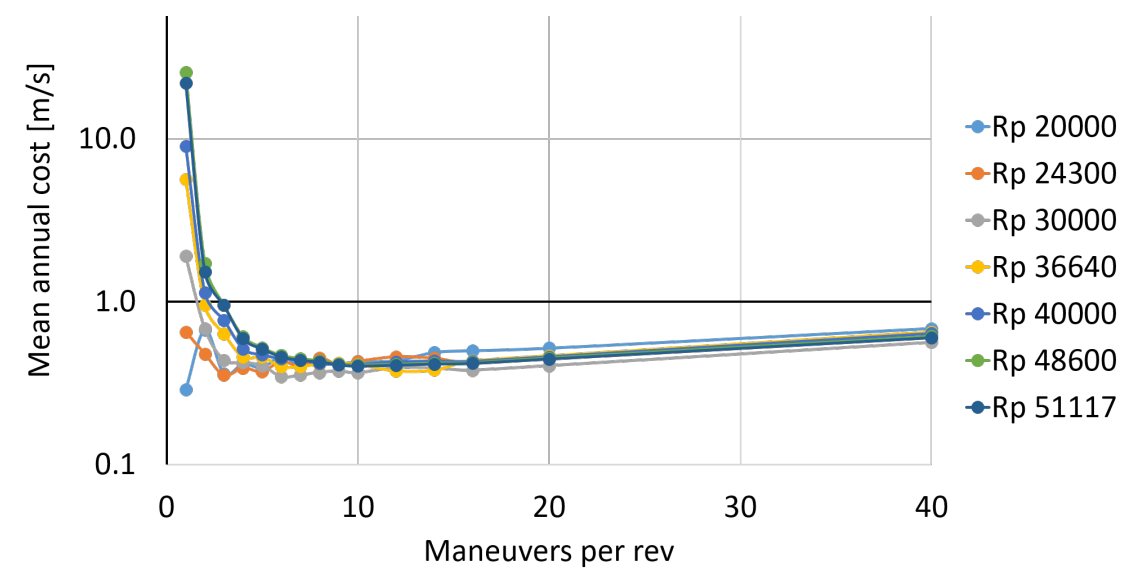

Fig. 14 Mean annual cost for selected unstable halo orbits with different number of maneuvers per revolution 


\section{Trend along halo family}

Halo orbits include orbits that are stable, i.e., NRHOs, as well as orbits that are unstable in nature. The station-keeping characteristics of these orbits are invariably different. The performance of the station-keeping algorithm on these orbits are presented in Figures 15a and 15b. Figure 15a describes the minimum cost for station-keeping, Figure $15 \mathrm{~b}$ represents the corresponding number of maneuvers per revolution required for minimal station-keeping case. For stable NRHOs, the mean annual cost for orbit maintenance is lower than that of unstable halo orbits in general. For NRHOs with perilune radius between $3200 \mathrm{~km}$ and $12,136 \mathrm{~km}$, the mean annual cost is between $0.05 \mathrm{~m} / \mathrm{s}$ to $0.13 \mathrm{~m} / \mathrm{s}$, while for unstable halo orbits the value increases to 0.35 and $0.45 \mathrm{~m} / \mathrm{s}$ for perilune radius between $24,300 \mathrm{~km}$ to $51,117 \mathrm{~km}$, respectively. For perilune radius between $12,136 \mathrm{~km}$ to $20,000 \mathrm{~km}$, close to the boundary between NRHO and unstable halo orbits, a transition zone can be observed where the mean cost gradually increases. Figure $15 \mathrm{~b}$ describes that for stable NRHO orbits, one maneuver per orbit executed at the apoapsis region provides the minimal station-keeping cost. The number of maneuvers for minimum mean cost gradually increases over the transition region close to the boundary between NRHO and unstable halo orbits. For highly unstable orbits a minimum of 10 maneuvers per revolution seems to be the most appropriate for minimum mean annual orbit maintenance cost. As expected, since the orbits are unstable and trajectories in the vicinity of these limit cycles are prone to divergence, more maneuvers are required to efficiently maintain the spacecraft in the nearby vicinity.

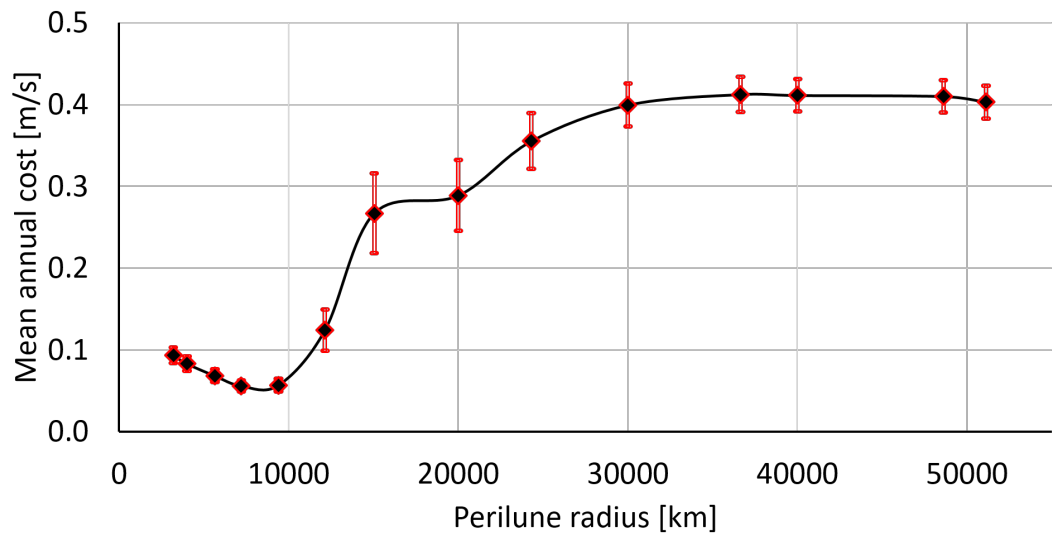

(a) Minimum annual station-keeping cost for different halo orbits with varying perilune radii. Mean and 1-sigma values are plotted.

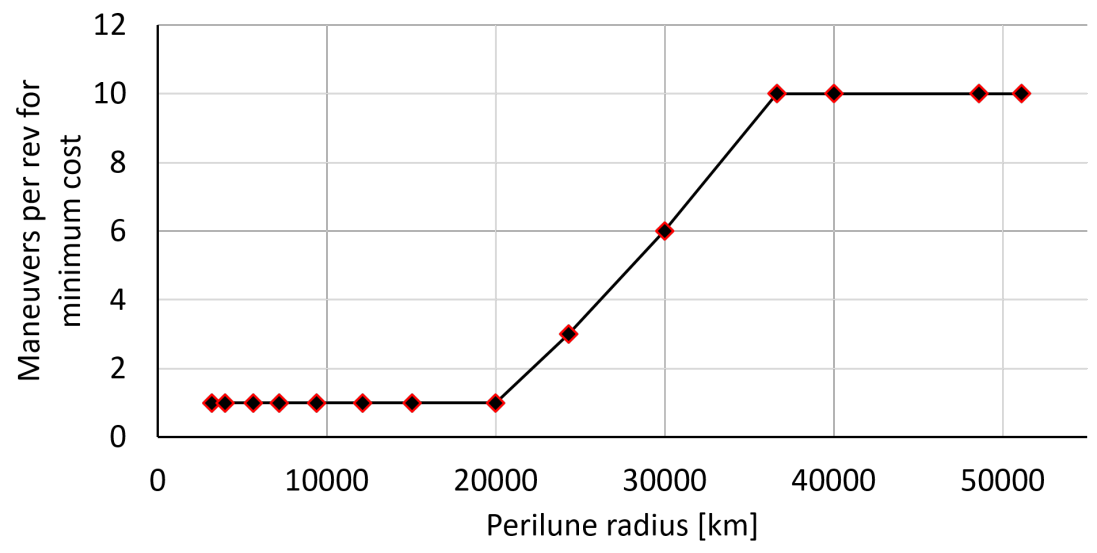

(b) Number of maneuvers per revolution for minimum annual station-keeping cost.

Fig. 15 Minimum annual station-keeping cost and corresponding number of maneuvers per revolution for Earth-Moon L2 southern halo family.

The distribution of maneuvers as well as the deviation of the spacecraft with respect to the reference trajectory along the NRHO orbits and the unstable halo orbits are distinct. Figures $16 \mathrm{a}$ and $16 \mathrm{~b}$ show the maneuver and deviation history 
for the case with 10 maneuvers along each orbit for an NRHO with perilune radius of 4000km; Figures $17 \mathrm{a}$ and $17 \mathrm{~b}$ show results for the same case with 10 maneuvers along an unstable halo orbit orbit with perilune radius of $40000 \mathrm{~km}$. Due to close proximity to the Moon, sharp peaks are observed for both the maneuver and deviation history in the case of the NRHO near the periapsis region, while values are very low near apoapsis region. In contrast the dynamic behavior near periapsis region is not very distinct to that near apoapsis region for an unstable halo orbit that is sufficiently far from the moon, therefore a gradual amplitude change is observed in control maneuver and deviation history.

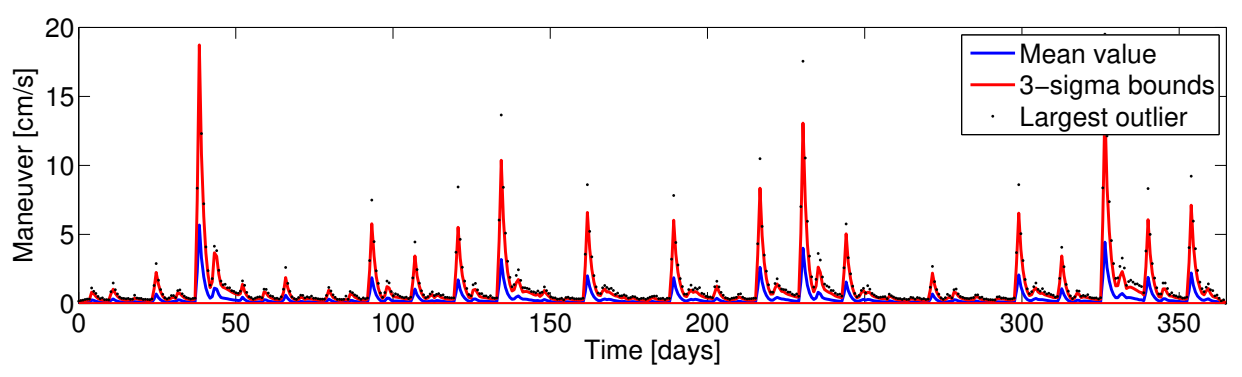

(a) Maneuver magnitude vs time

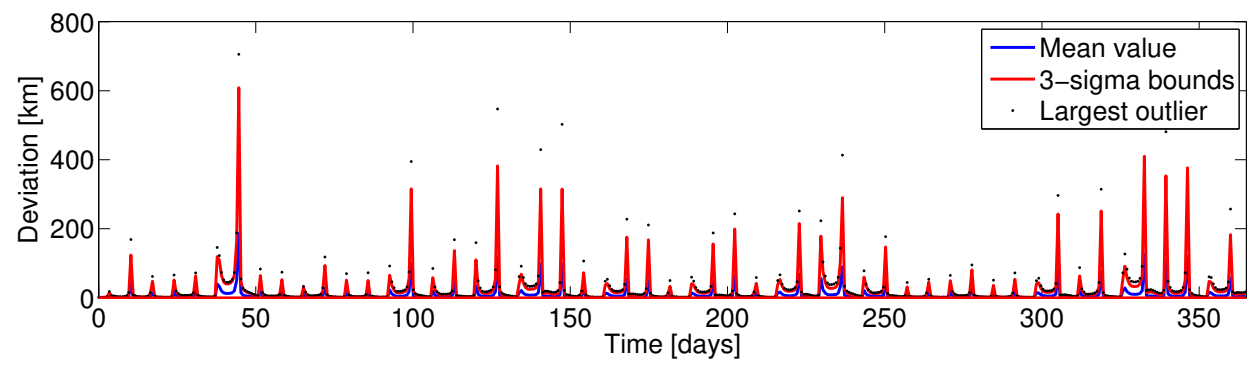

(b) Deviation vs time

Fig. 16 Maneuver magnitude and position deviation for NRHO with perilune radius $4000 \mathbf{k m}$ with $r=10^{14}$. Station-keeping maneuvers performed 10 times per revolution.

\section{E. Transfer cost}

For the 4:1 sidereal resonant NRHO with perilune radius $4000 \mathrm{~km}$, we obtained a transfer cost of $8.45 \mathrm{~m} / \mathrm{s}$ for one rev transfer with $3.71 \mathrm{~m} / \mathrm{s}$ at departure and $4.74 \mathrm{~m} / \mathrm{s}$ at arrival. Optimal values obtained were found to be independent of the number of intermediate patch points selected. The number of intermediate arcs, tested between 1 and 10, a minimum fuel transfer was obtained for 6 rev at $6.63 \mathrm{~m} / \mathrm{s}: 1.13 \mathrm{~m} / \mathrm{s}$ at departure and $5.50 \mathrm{~m} / \mathrm{s}$ at arrival. Note that the values only correspond to this particular orbit, and a different choice of orbit/trajectory may result in a different cost. The method is however independent of the new arc and is valid for transfer between other orbits as well. A detailed cost analysis for different length of transfer arc is provided in Figure 18. A geometric representation of transfer arc for 1 rev transfer through a $4 \mathrm{rev}$ transfer is pictured in Figure 19 . The same procedure can be applied to transfer between other NRHO, unstable halo orbits, and others.

\section{F. STK results}

We used Systems Tool Kit (STK) to validate our station-keeping results. STK is a software package that provides high-fidelity space dynamics simulation. Similar to the procedure adopted earlier, a zero-cost reference trajectory was generated using multiple shooting scheme with trajectories propagated through STK. To incorporate effects of J2 zonal harmonics of the Moon seperate from Earth gravity, Sun gravity and the solar radiation pressure, the Moon HPOP Default v10 propagator was used in STK. We tested our station-keeping algorithm on this baseline solution. Observation and estimation of states using a Kalman filter were done by passing spacecraft data to MATLAB as discussed earlier, beyond which the necessary Gain matrices were computed. About 100 Monte Carlo simulations were performed in STK to determine the mean annual station-keeping cost. For the 4:1 sidereal resonant NRHO with $4000 \mathrm{~km}$ perilune radius propagated for one year time span, the results showed an annual mean cost of $9.60 \mathrm{~cm} / \mathrm{s}$ with a standard deviation of the 


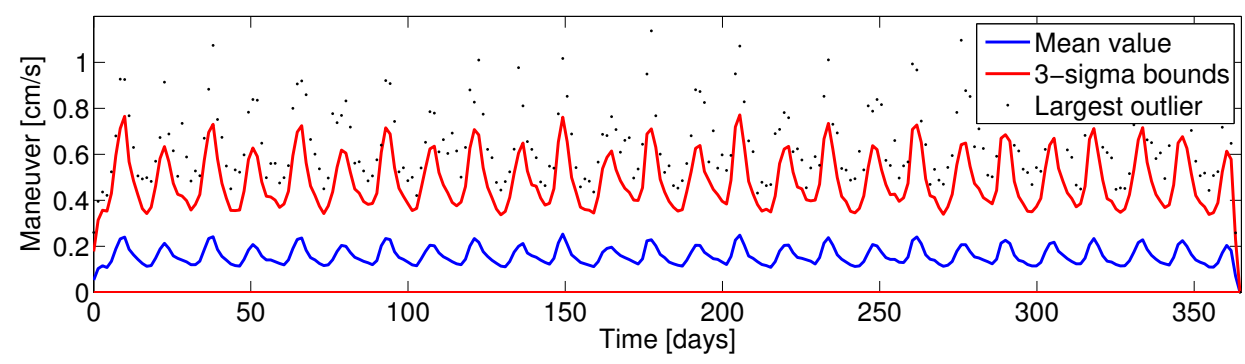

(a) Maneuver magnitude vs time

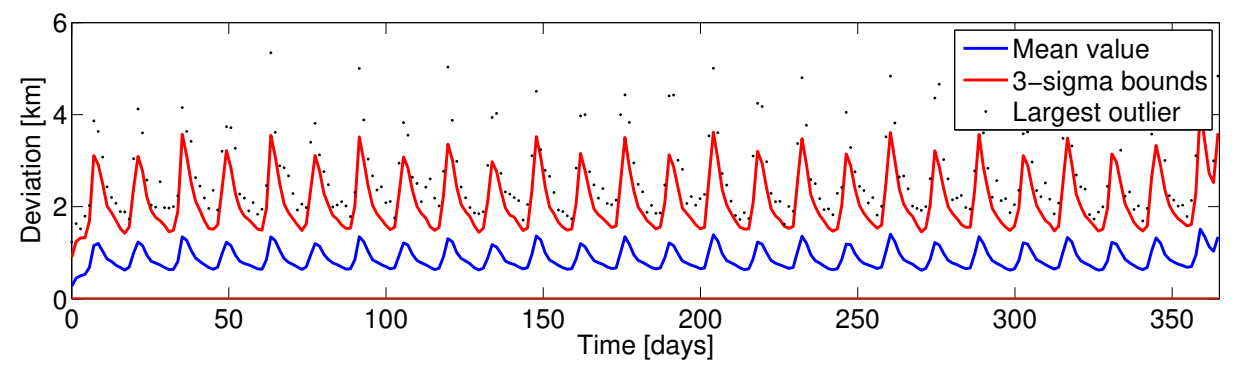

(b) Deviation vs time

Fig. 17 Maneuver magnitude and position deviation for unstable halo orbit with perilune radius $40000 \mathbf{~ k m}$ with $r=10^{14}$. Station-keeping maneuvers performed 10 times per revolution.

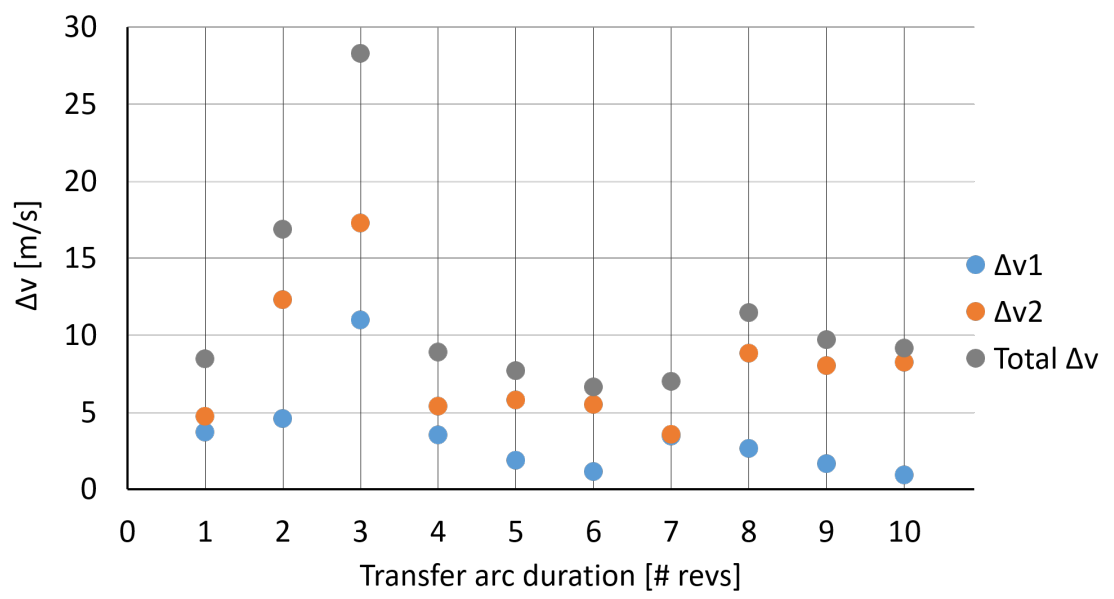

Fig. 18 Transfer cost to transition from one long horizon reference trajectory to another. Values shown here are to transition from one baseline of perilune radius $4000 \mathrm{~km}$ NRHO to another NRHO with the same perilune radius for an extended mission scenario.

recorded observations of $1.16 \mathrm{~cm} / \mathrm{s}$. The values are consistent with those obtained previously: a mean of $8.34 \mathrm{~cm} / \mathrm{s}$ and standard deviation of $0.86 \mathrm{~cm} / \mathrm{s}$.

\section{Conclusions}

We present a potential method for station-keeping for the halo family of orbits in the Earth-Moon system. The method consists of a two-stage procedure: firstly to overcome non predictable perturbations due to navigational errors and unmodeled forces that may exist in the system. For this, we implemented a control scheme along with an observer, specifically a Kalman filter. The control gain was computed as a solution to the LQR problem, whose penalty was chosen based on the covariance of the state estimation. This was done to ensure that the algorithm causes higher 


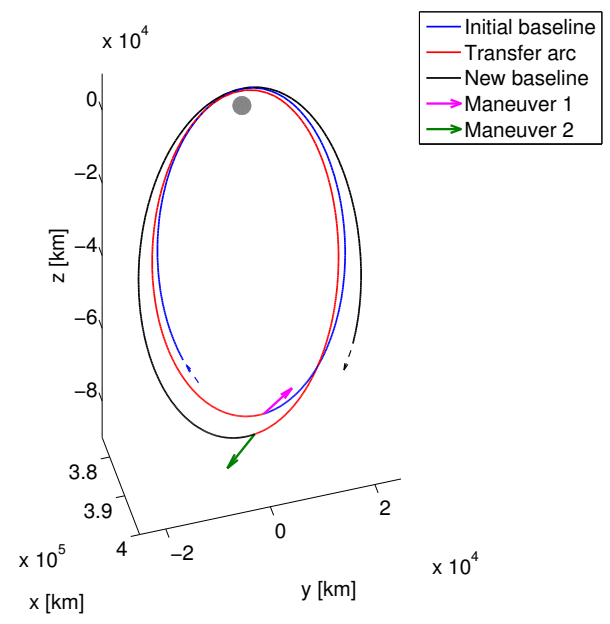

(a) 1 rev transfer arc

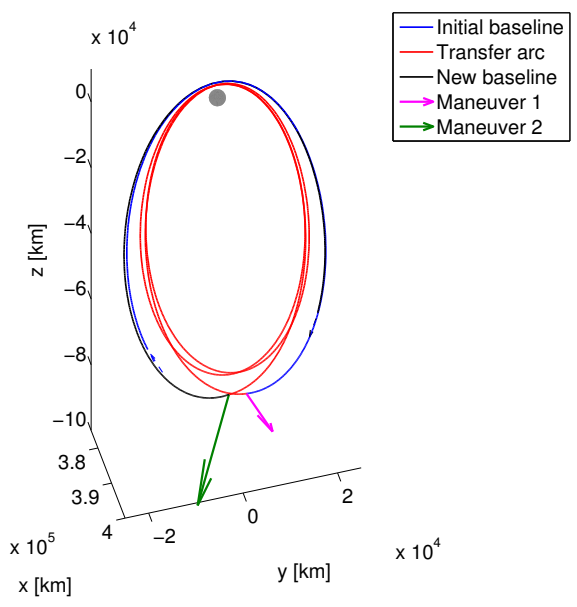

(c) 3 rev transfer arc

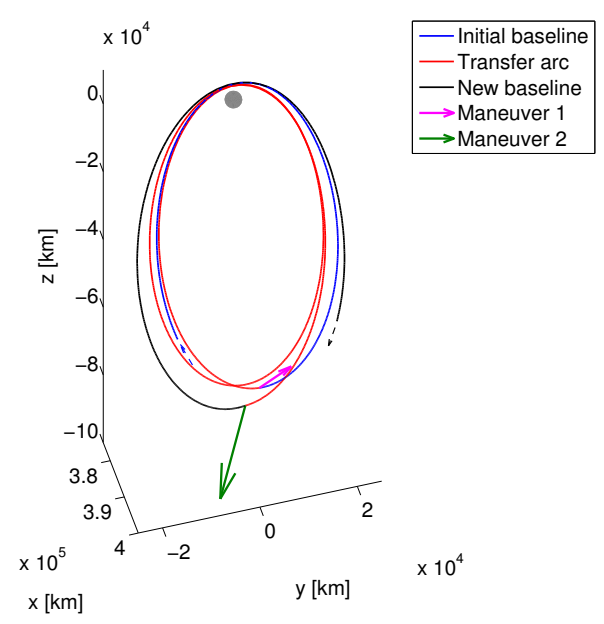

(b) 2 rev transfer arc

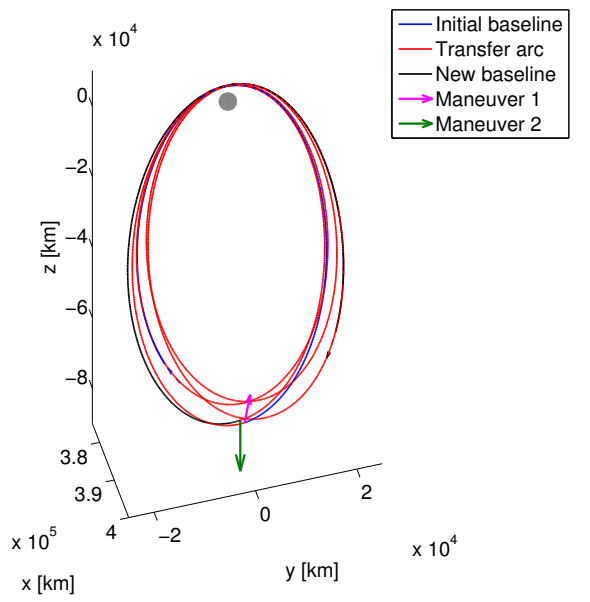

(d) 4 rev transfer arc

Fig. 19 Optimized transfer arc for different duration of transfer. Only a small section of the initial and the new baseline trajectory are plotted for visual convenience.

penalties resulting in smaller maneuvers in regions of higher uncertainty. Using this strategy, the algorithm successfully avoids alteration in states in regions of lower confidence, therefore any errors in state coordinates may not amplify rapidly upon propagating downstream. By thorough analysis, we demonstrated that this technique works effectively for both stable NRHO and other unstable halo orbits.

The second part of the procedure implements a minimum-fuel orbital-transfer controller for long-horizon maneuvers. This is done because, due to various reasons, a reference orbit cannot be expected to last indefinitely. Hence, we provide a method that hops from one orbit to another orbit as an alternative choice. The results showed adequate, indefinite tracking of NRHOs and fuel consumption within accepted standards.

\section{Appendix}

In Table 3 and Figure 20, we show selected halo orbits and their specifications. Note that the stability index in the table are given by $\frac{1}{2}\left(\lambda+\frac{1}{\lambda}\right)$, where $\lambda$ is the largest magnitude of eigenvalue of the monodromy matrix. 
Table 3 Specifications of Earth-Moon southern L2 halo orbits

\begin{tabular}{|c|c|c|c|}
\hline Rp [km] & Characteristics & Subclassification & Stability index \\
\hline 3200 & $9: 2$ synodic resonance & NRHO & -1.312 \\
4000 & $4: 1$ sidereal resonance & NRHO & -1.441 \\
5645 & $4: 1$ synodic resonance & NRHO & -1.617 \\
7200 & $7: 2$ sidereal resonance & NRHO & -1.689 \\
9400 & $7: 2$ synodic resonance & NRHO & -1.632 \\
12136 & $3: 1$ sidereal resonance & NRHO & -1.280 \\
15038 & $3: 1$ synodic resonance & NRHO & -0.691 \\
20000 & $5: 2$ sidereal resonance & & 2.926 \\
24300 & $5: 2$ synodic resonance & & 8.665 \\
30000 & - & & 25.498 \\
36640 & $2: 1$ sidereal resonance & & 77.661 \\
40000 & - & & 132.649 \\
48600 & $2: 1$ synodic resonance & & 452.025 \\
51117 & almost flat & & 605.994 \\
\hline
\end{tabular}

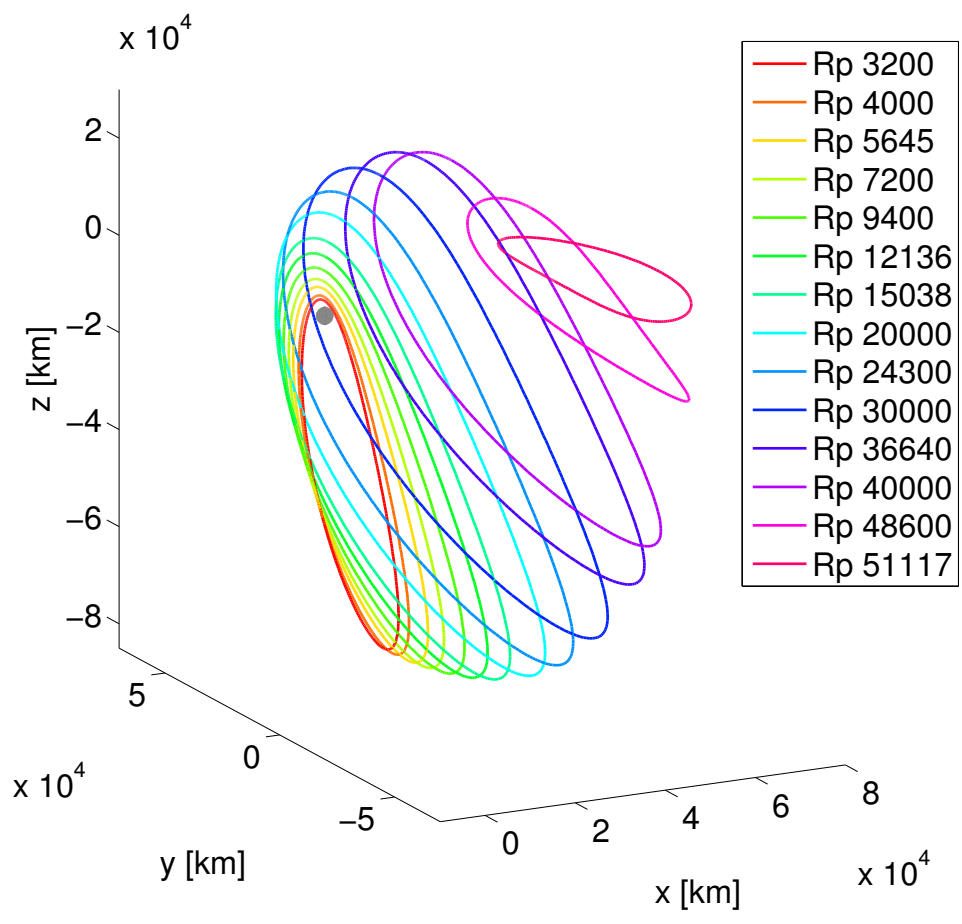

Fig. 20 Selected orbits from Earth-Moon L2 southern halo family

\section{References}

[1] Laurini, K. C., Hufenbach, B., Hill, J., and Ouellet, A., "The global exploration roadmap and expanding human/robotic exploration mission collaboration opportunities," Proc. Int. Astronautical Congress, Jerusalem, 2015, IAC-15.A3.1.1.

[2] Davis, D., Bhatt, S., Howell, K., Jang, J.-W., Whitley, R., Clark, F., Guzzetti, D., Zimovan, E., and Barton, G., “Orbit maintenance and navigation of human spacecraft at cislunar near rectilinear halo orbits," Proc. AAS/AIAA Astrodynamics Specialist Conf., Stevenson, WA, 2017, AAS 17-269. 
[3] Thornton, C. L., and Border, J. S., Radiometric Tracking Techniques for Deep-Space Navigation, Wiley, Hoboken, NJ, 2003.

[4] Howell, K. C., and Gordon, S. C., "Orbit determination error analysis and a station-keeping strategy for Sun-Earth L1 libration point orbits,” J. Astronaut. Sci., Vol. 42, No. 2, 1994, pp. 207-228.

[5] Muralidharan, V., "Orbit Maintenance Strategies for Sun-Earth/Moon Libration Point Missions: Parameter Selection for Target Point and Cauchy-Green Tensor Approaches,” Master's thesis, Purdue University, West Lafayette, IN, 2017.

[6] Guzzetti, D., Zimovan, E. M., Howell, K. C., and Davis, D. C., "Stationkeeping analysis for spacecraft in lunar near rectilinear halo orbits," AAS/AIAA Space Flight Mechanics Meet., San Antonio, 2017, 17-395.

[7] Lei, L., and Chunyang, H., "Scheme design of the CHANG'E-5T1 extended mission," Chinese Journal of Aeronautics, Vol. 31, No. 7, 2018, pp. 1559-1567.

[8] Woodard, M., Folta, D., and Woodfork, D., "ARTEMIS: the first mission to the lunar libration orbits," 21 st International Symposium on Space Flight Dynamics, Toulouse, France, 2009.

[9] Pavlak, T. A., “Trajectory design and orbit maintenance strategies in multi-body dynamical regimes," Ph.D. thesis, Purdue University, West Lafayette, IN, 2013.

[10] Battin, R. H., and Levine, G. M., "Application of Kalman filtering techniques to the Apollo program(Kalman filtering techniques applied to coasting flight navigation problems of Apollo lunar mission)," 1970., 1970, pp. 335-361.

[11] Grewal, M. S., and Andrews, A. P., "Applications of Kalman filtering in aerospace 1960 to the present [historical perspectives]," IEEE Control Systems Magazine, Vol. 30, No. 3, 2010, pp. 69-78.

[12] Woodard, M., Cosgrove, D., Morinelli, P., Marchese, J., Owens, B., and Folta, D., "Orbit determination of spacecraft in Earth-Moon L1 and L2 libration point orbits," 2011.

[13] Montenbruck, O., and Gill, E., Satellite orbits: models, methods and applications, Springer Science \& Business Media, 2012.

[14] Simon, D., Optimal state estimation: Kalman, H infinity, and nonlinear approaches, John Wiley \& Sons, 2006.

[15] Folta, D., Woodard, M., and Cosgrove, D., "Stationkeeping of the first earth-moon libration orbiters: The artemis mission," 2011.

[16] Folta, D., and Sweetser, T., “ARTEMIS mission overview: from concept to operations," 2011.

[17] Baker, D., Sears, N., Suomals, J., and White, R., "Lunar Orbit Determination by Star Occultation and MSFN Tracking," Massachusetts Institute of Technology Instrumentation Lab., Rept. E-1429 (September 1963), Vol. 68, 1963.

[18] Floberghagen, R., Visser, P., and Vasile, M., "Low lunar orbit analysis, determination and selection for soft landing on the lunar South Pole," Space Flight Dynamics, Vol. 403, 1997, p. 321.

[19] Mazarico, E., Rowlands, D., Neumann, G., Smith, D., Torrence, M., Lemoine, F., and Zuber, M., "Orbit determination of the lunar reconnaissance orbiter," Journal of Geodesy, Vol. 86, No. 3, 2012, pp. 193-207.

[20] Slojkowski, S. E., "Lunar Reconnaissance Orbiter orbit determination accuracy analysis,” 2014.

[21] Songhe, Q., Yong, H., Peijia, L., Quan, S., Min, F., Xiaogong, H., and Guangli, W., "Orbit and Tracking Data Evaluation of Chang'E-4 Relay Satellite," Advances in Space Research, 2019.

[22] Stengel, R. F., Optimal Control and Estimation, $2^{\text {nd }}$ ed., Dover, Mineola, NY, 1994. 